\title{
Vezatin Is Essential for Dendritic Spine Morphogenesis and Functional Synaptic Maturation
}

\author{
Lydia Danglot, ${ }^{1,2}$ Thomas Freret, ${ }^{3}$ Nicolas Le Roux, ${ }^{4,5}$ Nicolas Narboux Nême, ${ }^{4,5}$ Andrea Burgo, ${ }^{1,2}$ Vincent Hyenne, ${ }^{6,7}$ \\ Anne Roumier, ${ }^{4,5}$ Vincent Contremoulins, ${ }^{1,8}$ François Dauphin, ${ }^{3}$ Jean-Charles Bizot, ${ }^{9}$ Guilan Vodjdani, ${ }^{10,11}$ \\ Patricia Gaspar, ${ }^{4,5}$ Michel Boulouard, ${ }^{3}$ Jean-Christophe Poncer, ${ }^{4,5}$ Thierry Galli, ${ }^{1,2}$ and Marie-Christine Simmler ${ }^{1,2}$ \\ ${ }^{1}$ Centre national de la recherche scientifique (CNRS) UMR7592, Université Paris Diderot, Sorbonne Paris Cité, Institut Jacques Monod, 75205 Paris, France, ${ }^{2}$ Institut \\ National de la Santé et de la Recherche Médicale (INSERM) ERL U950, 75205 Paris, France, ${ }^{3}$ Université Caen Basse-Normandie, EA4259, 14032 Caen, France, \\ ${ }^{4}$ INSERM UMR839, Institut du Fer à Moulin, 75005 Paris, France, ${ }^{5}$ Université Pierre et Marie Curie, Sorbonne University, Paris, France, ${ }^{6} \mathrm{CNRS}$ UMR7104, \\ Université de Strasbourg, Institut de Génétique et Biologie Moléculaire et Cellulaire (IGBMC), 67404 Illkirch, France, ${ }^{7}$ INSERM U964, IGBMC, 67404 Illkirch, France, \\ ${ }^{8}$ ImagoSeine, Institut Jacques Monod, 75205 Paris, France, ${ }^{9}$ Key-Obs SA, Parc Technologique, 45100 Orléans, France, ${ }^{10}$ CNRS UMR7225 Université Pierre et Marie \\ Curie, Sorbonne University, Centre de Recherche de l'Institut du Cerveau et de la Moelle épinière (CRICM), GH Pitié-Salpetrière, 75013 Paris, France, and ${ }^{11}$ INSERM \\ UMRS975, Université Pierre et Marie Curie, Sorbonne University, CRICM, GH Pitié-Salpétrière, 75013 Paris, France
}

Vezatin is an integral membrane protein associated with cell-cell adhesion complex and actin cytoskeleton. It is expressed in the developing and mature mammalian brain, but its neuronal function is unknown. Here, we show that Vezatin localizes in spines in mature mouse hippocampal neurons and codistributes with PSD95, a major scaffolding protein of the excitatory postsynaptic density. Forebrainspecific conditional ablation of Vezatin induced anxiety-like behavior and impaired cued fear-conditioning memory response. Vezatin knock-down in cultured hippocampal neurons and Vezatin conditional knock-out in mice led to a significantly increased proportion of stubby spines and a reduced proportion of mature dendritic spines. PSD95 remained tethered to presynaptic terminals in Vezatindeficient hippocampal neurons, suggesting that the reduced expression of Vezatin does not compromise the maintenance of synaptic connections. Accordingly, neither the amplitude nor the frequency of miniature EPSCs was affected in Vezatin-deficient hippocampal neurons. However, the AMPA/NMDA ratio of evoked EPSCs was reduced, suggesting impaired functional maturation of excitatory synapses. These results suggest a role of Vezatin in dendritic spine morphogenesis and functional synaptic maturation.

\section{Introduction}

The brain has a tremendous capacity for adaptive modifications during both development and adulthood. This neuronal plasticity is crucial for learning and memory. For instance, in Fmr1-null

\footnotetext{
Received June 17, 2011; revised May 10, 2012; accepted May 16, 2012.

Author contributions: L.D., P.G., and M.-C.S. designed research; L.D., T.F., N.L.R., N.N.-N., A.B., V.H., A.R., G.V., M.B., J.C.P., and M.-C.S. performed research; L.D., V.C., F.D., and M.-C.S. contributed unpublished reagents/analytic tools; L.D., T.F., J.-C.B., M.B., J.C.P., and M.-C.S. analyzed data; L.D., P.G., J.C.P., T.G., and M.-C.S. wrote the paper.

This work was supported by the Fédération de la Recherche sur le Cerveau (M.C.S.), the Association Française contre les Myopathies, the Marie de Paris Medical Research and Health Program, the Fondation pour la Recherche Médicale, and the Ecole des Neurosciences de Paris (T.G.). We thank Dr Günther Schütz (Cancer Research Center, Heidelberg, Germany) for the CamKIl $\alpha$ cre transgenic mice and Dr Stephan Krauss (Institute for Microbiology, 0slo, Norway) for the D6cre transgenic mice; Drs Martin Holzenberger (INSERM U893, Paris, France) and Alessandra Pierani (IJM, Paris, France) for providing adult CamKIlcre and D6cre mice, respectively; Ondrej Machon (Institute of Molecular Genetics, Prague, Czech Republic) for sharing information on the D6cre line; Marie-José Lecomte for Lentivirus-cre supernatant (CRICM, Paris, France); Gérald Née (Université de (aen Basse Normandie, France), Sabrina David, and Alexandre Herpin (Key-Obs SA, Orléans, Paris) for great technical help; Véronique Agin, Pauline Obiang, and Denis Vivien for providing the fear conditioning system (Université de Caen Basse-Normandie, France); Xavier Baudin, Lamia Baba-Aissa, and Aude Jobart-Malfait (ImagoSeine, IJM, Paris, France) for constant support in imaging and software manipulations; Dr Marika Nosten-Bertrand (INSERM U513, Paris, France) for enthusiastic help and discussions, and Kathleen Zylbersztejn and Thierry Galli's group for support and criticisms. We are especially grateful to Professor Christine Petit (Institut Pasteur, INSERM UMRS587, Collège de France, Paris, France) for critical reading of the manuscript.

The authors declare no competing financial interests.

Correspondence should be addressed to Marie-Christine Simmler, Institut Jacques Monod, 15 rue Hélène Brion, 75205 Paris, France. E-mail: simmler.marie-christine@ijm.univ-paris-diderot.fr.

DOI:10.1523/JNEUROSCI.3084-11.2012

Copyright $\odot 2012$ the authors $\quad 0270-6474 / 12 / 329007-16 \$ 15.00 / 0$
}

mice, an experimental model for intellectual disabilities associated with fragile $\mathrm{X}$ syndrome, the learning deficit is most likely due to alteration in synaptic plasticity (Comery et al., 1997; Nimchinsky et al., 2001; Cruz-Martin et al., 2010). Deficits in neuronal circuit reorganization are also thought to be responsible for age-related cognitive decline as aged humans or animals show dysfunctions in the mechanisms of synaptic efficacy (Burke and Barnes, 2006).

Dendritic spines are critical sites of excitatory synaptic contact, signal integration, and functional dynamics (Sheng and Kim, 2002). During development, spine morphology changes as a result of glutamate receptor activity (Hering and Sheng, 2001) and actin polymerization (Lin et al., 2005; Kerr and Blanpied, 2012) with acquisition/densification of AMPA receptors (AMPARs), which allow for glutamate to activate otherwise silent synapses with only NMDA receptors (NMDARs) (Kerchner and Nicoll, 2008). It was shown that dysfunctions in the development/dynamics of dendritic spines are associated with many neurodevelopmental diseases (Bourgeron, 2009; von Bohlen and Halbach, 2010). The molecular mechanisms implicated in the formation/maturation of synaptic contacts in cognitive processing are thus central to the normal and pathological synaptic physiology (Segal, 2005; Penzes et al., 2011).

Dendritic spines are dynamically connected to presynaptic terminals via adhesion complexes crucial for synaptogenesis and 
spine stabilization (Takeichi and Abe, 2005; Sandi and Bisaz, 2007; Shapiro et al., 2007; Benson and Huntley, 2011). Vezatin, a ubiquitous integral protein, is indirectly associated with E-cadherin-catenin complex and actin cytoskeleton (KusselAndermann et al., 2000; Bahloul et al., 2009). Its functions have mainly been explored in epithelial cells. Loss of Vezatin is progressively detrimental to embryonic development (Hyenne et al., $2005,2007 \mathrm{~b}$ ), and Vezatin is critical for preserving the integrity of the cellular junctions during long-term mechanical stress occurring at the level of inner ear hair cells in response to sound. Moreover, the inner ear-specific Vezatin conditional invalidation leads to progressive deafness (Bahloul et al., 2009). We previously observed that Vezatin is highly expressed in the brain (KusselAndermann et al., 2000; Hyenne et al., 2007a), where its function is unknown. Here, we show that Vezatin is enriched in dendritic spines in mouse hippocampal neurons. Using Vezatin knockdown and conditional knock-out before (D6cre) or after

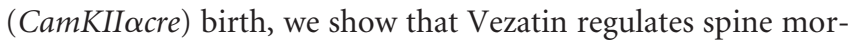
phology, morphological changes are not associated with compromised synaptic contacts, but postsynaptic Vezatin plays a critical role in the morpho-functional maturation of excitatory postsynaptic elements.

\section{Materials and Methods}

Animals. Homozygous 129/SvxC57BL/6Pas Vezatin flox/flox (Vezatin $^{\text {tmlSmc }}$ ) (Hyenne et al., 2007b) were crossed to the 129/Sv PGK-1-cre transgenic line, which expresses partial recombinase activity, to remove specifically the floxed neomycin cassette and leave intact the floxing of Vezatin exon5 (EMMA, EM:01817 or Vezt flox5/flox5). This line will be named hereafter $V e z t^{f l f l}$. To generate conditional Vezatin-deficient mice, Vezt $t^{f l f l}$ were crossed with transgenic mice that express cre recombinase under the control of the calcium/calmodulin-dependent kinase II $\alpha$ subunit of the CamKII promoter (Casanova et al., 2001). Cre recombinase is expressed in the DG and CA1/CA3 regions of the hippocampus, cortex, olfactory bulb, and amygdala and, more weakly, in the striatum, thalamus, and hypothalamus. The CamKIIcre2 line was backcrossed on a C57BL/6J genetic background by Dr. Martin Holzenberger (INSERM U893, Hopital Saint-Antoine, Paris, France). Crosses were systematically between female homozygous for the Vezt-floxed allele and carrying the cre transgene and homozygous male for the Vezt-floxed allele, giving rise to progeny among which $50 \%$ carried the CamKIIcre transgene (Vezt $t^{f l f f}::$ CamKIIcre + or CamKIIcKO) and $50 \%$ did not (Vezt $t^{f l f l}$ or control). Vezt $t^{f l f l}$ were also crossed with transgenic D6cre mice that express Cre recombinase under the control of an enhancer element derived from the mouse Dach1 promoter (D6), active in the neocortex and the hippocampus. D6cre line was described by van den Bout et al. (2002), who showed Cre recombinase expression early during development [embryonic day (E) 10.5]. Expression was restricted at later embryonic stages to hippocampus (the DG and CA1/CA3 regions in adult mice) and cortex. Crosses were as for the CamKIIcre line and were named Vezt $t^{f l f l}:: D 6 c r e+$ $(D 6 \mathrm{cKO})$ and $V e z t^{f l / f l}$ (control). PCR genotyping was carried out on tails of individual embryos or adult mice with primers cre1 and cre2 (Hyenne et al., 2007b). All in vivo experiments in the present study were performed on male mice on a randomized $129 / \mathrm{Sv}$ and C57BL/6J genetic background. Animals were weaned at 4 weeks and housed two to six per cage by sex and litter regardless of the genotype under standard conditions, with food and water available ad libitum. Vezt fl/null mice (EMMA, EM: 04326) carrying one floxed and one null Vezatin allele (Hyenne et al., 2007b) were used for control of antibody specificity. Experiments were performed in accordance with the European Communities Council Directive regarding the care and use of animals for experimental procedures (2010/63/UE) and were approved by the local ethical committee.

Preparation of synaptosomes (P2). Hippocampi from 2- to 4-monthold males were homogenized in homogenization buffer ( 5 mM HEPES, $\mathrm{pH} 7.4,320 \mathrm{~mm}$ sucrose) containing a protease inhibitor mixture (Roche Applied Science). Nuclei and cellular debris were removed twice by $1000 \times \mathrm{g}$ centrifugation. The supernatant was spun at $16,000 \times \mathrm{g}$ for 60 min at $4^{\circ} \mathrm{C}$, resulting in S2 and P2 fractions. Aliquots were subjected to SDS-PAGE analysis by using NuPAGE $4-12 \%$ Bis-Tris or Tris-Acetate miniGels (Applied Biosystems), gradient gels and the manufacturer's buffers, transferred onto nitrocellulose filters (Protran; Schleicher and Schuell) and then processed for Western blotting. Detection and relational quantification was carried out by the infrared imaging system Odyssey (LI-COR Biosciences). Blots were quantified using ImageJ software (National Institutes of Health, Bethesda, MD) and mouse anti- $\beta$-tubulin at 1:5000 (Hybridoma Bank, University of Iowa, IA) or anti-GAPDH (Mab374), 1:300 (Millipore, Merck Millipore) antibodies.

RT-PCR analysis. Proper exon 5 excision following cre recombination was tested by RT-PCR analysis using hippocampus mRNA from 5-weekold control and CamKIIcKO (or D6cKO) male mice, as described by Bahloul et al. (2009).

Primary neuronal culture, immunostaining, and confocal analysis of spines. Culture and immunostaining of hippocampal neurons from E18.5 mouse embryos were adapted from Danglot et al. (2003). Hippocampi were dissociated by treatment with trypsin $(0.25 \%$ for 15 min at $37^{\circ} \mathrm{C}$ ) followed by trituration with a constricted Pasteur pipette. The cells were plated onto poly-ornithine-coated coverslips $(1 \mathrm{mg} / \mathrm{mL})$ (Sigma-Aldrich) in 4-well tissue culture plates at a density of $2 \times 10^{4}$ cells/well in astrocyte maintenance medium [modified Eagle's medium, $10 \%$ horse serum, $0.06 \%$ glucose, $100 \mathrm{U} / \mathrm{mL}$ penicillin, and $100 \mu \mathrm{g} / \mathrm{mL}$ streptomycin (MEM-HS)]. After $4 \mathrm{~h}$, when the cells were attached to the substrate, the tissue culture plate medium was changed and maintained in Neurobasal-B27 glia-conditioned growth medium (Gibco Applied Biosystems) containing 2\% B27 supplement (Gibco), and $500 \mu \mathrm{M}$ L-glutamine (Sigma-Aldrich). Neurobasal B27 glia medium was conditioned by incubation of at least $18 \mathrm{~h}$ on a confluent layer of astrocytes prepared from cerebral hemispheres of neonatal mouse pups, maintained in culture in MEM-HS up to 6 months. The conditioning growth medium was changed every $7 \mathrm{~d}$. Cultures were incubated at $37^{\circ} \mathrm{C}$ in a humidified atmosphere of $95 \%$ air and $5 \% \mathrm{CO}_{2}$. Neurons were fixed with cold methanol for $5 \mathrm{~min}$ at $-20^{\circ} \mathrm{C}$ to better preserve cytoskeletal structures. Fixation with $4 \%$ paraformaldehyde for $20 \mathrm{~min}$ at room temperature (RT) was used when phalloidin staining was examined. Quenching with $\mathrm{NH} 4 \mathrm{Cl}$ for $15 \mathrm{~min}$ was followed by a permeabilization step for $4 \mathrm{~min}$ with a mixture of $0.1 \%$ Triton X-100/PBS/0.125\% cold water fish skin gelatin (gelatin) (Sigma-Aldrich). After three PBS $\times 1$ washings, neurons were incubated in blocking solution containing PBS $/ 0.25 \%$ gelatin for 30 min. Immunocytological staining was performed by incubation with the primary antibody in $\mathrm{PBS} / 0.125 \%$ gelatin overnight at $4^{\circ} \mathrm{C}$, followed by incubation in the secondary antibody in PBS/0.125\% gelatin for $45 \mathrm{~min}$ at room temperature. Confocal images for mushroom/stubby spine 3D reconstruction were obtained using a confocal microscope (TCS SP5; Leica Microsystems) and a $63 \times$ objective zoom 2.73 with sequential acquisition settings of $1024 \times 1024$ pixels and the Clipping Plane device in the Surpass Scene built-in tool from the Imaris software 7.0 package (Bitplane Scientific Software). Confocal images for spine colabeling were obtained using a confocal microscope (TCS SP2; Leica Microsystems) and a $63 \times$ objective with sequential acquisition settings of $512 \times 512$ pixels. Each image was a Z-series projection of 5-7 images, taken at 0.326 $\mu \mathrm{m}$ depth intervals. Size of pixels was $0.1 \times 0.1 \mu \mathrm{m}$. Quantification was performed on 17 individual spines at 14 DIV and 21 DIV. Colocalization was measured by color-separating PSD95/Vezatin channels using a threshold level for each channel identical for each group of neurons. We determined the number of pixels in each channel and the overlapping number and proportion of pixels in each channel using the colocalization analysis plug-in in the ImageJ software (National Institutes of Health). 3D spine reconstruction was carried out using Amira 4.3 software (Visage Imaging, San Diego, CA). Surface structure was obtained with the surface rendering tool. Quantification of pre-postsynaptic appositions was carried out using a MetaMorph (Molecular Devices) software device.

cDNA transfection and time-lapse imaging. Dissociated neurons from rat cortex (Wistar) were cultured and transfected at 7 DIV using LipofectAMINE 2000 and $1 \mu \mathrm{g}$ of plasmid DNA, as recommended by the manufacturer (Applied Biosystems). Vezatin-GFP was described by Hyenne et al. (2005). Monomeric RFP-actin was a generous gift of Dr. Elaine Fuchs (The Rockefeller University, New York, NY). After 16 h, neurons 
were imaged by time-lapse experiments. Dual-color channels were collected sequentially every $10 \mathrm{~s}$ with an inverted microscope (DMI6000B; Leica Microsystem) equipped with a high-pressure mercury arc lamp (HBO $100 \mathrm{~W}$; Osram), 63×/1.6 plan-apochromat oil-immersion objectives, and a digital camera (Cascade:512B; Roper Scientific). Imaging was conducted in modified Krebs-Ringer-HEPES buffer (140 mM NaCl, 2.8 mм KCl, 1 mм MgCl2, 2 mм CaCl2, 10 mм HEPES, 5.5 mм glucose, $\mathrm{pH}$ 7.4, with $\mathrm{NaOH}$ ) in a temperature-controlled chamber. Lamp power and exposure time were the lowest possible (10-30\% Hg lamp, 50-100 ms) to avoid phototoxicity.

Lentiviral vector construction, production, and neuron transduction. The backbone of the lentiviral plasmid, $p$ Trip, has been previously described (Zennou et al., 2001). It is deleted for the HIV-1 enhancer and promoter sequences in the U3 region of the 3'LTR (Sirven et al., 2000). The nlsCre DNA fragment [kindly provided by Dr. François Tronche (Kellendonk et al., 1996)] was inserted in the $p$ Trip $\Delta$ U3.Pgk-WPRE vector, such that the Cre gene fused to a nuclear localization sequence is placed under the control of the murine Pgk phosphoglycerate kinase promoter [kindly provided by Dr. Alexis-Pierre Bemelmans (Bemelmans et al., 2005)], followed by the WPRE (Woodchuck hepatitis virus Posttranscriptional Regulatory Element) sequence, known to enhance transgene long-term expression (Zufferey et al., 1997). The resulting construct, $p$ Trip $\Delta \mathrm{U} 3 . P g k$-nlsCre-WPRE, was used to produce the recombinant lentiviral vector stocks, LV-Pgk-nlsCre (referred to as LV-Cre hereafter). LV-Cre stocks were produced by the calcium phosphate cotransfection of $293 \mathrm{~T}$ cells with the $p 8.91$ encapsidation plasmid (Zufferey et al., 1997), the vesicular stomatitis virus glycoprotein-G-encoding $p H C M V-G$ plasmid (Yee et al., 1997), and the $p$ Trip $\Delta$ U3.Pgk-nlsCre-WPRE recombinant vector as previously described (Zennou et al., 2001). Supernatants were treated with DNase I (Roche Diagnostics ) before ultracentrifugation, and the resulting pellet was resuspended in PBS, distributed into aliquots, and frozen at $80^{\circ} \mathrm{C}$ until use. Each new LV-Cre stock was titered by quantification of the HIV p24 Gag antigen with an ELISA kit (Beckman Coulter; the p24 concentration from different viral stock productions averaged $5 \mathrm{ng} / \mu \mathrm{l}$ of p24) and by determining the transducing units per milliliter in vitro in a functional assay using the $\mathrm{CV} 1-5 \mathrm{~B}$ reporter cell line in which Cre-mediated recombination induces $\beta$-gal expression (Kellendonk et al., 1996). The values from one batch to another averaged $4.10^{6}$ $\mathrm{TU} / \mathrm{mL}$. E18.5 Vezt fl/f-dissociated hippocampal neurons were plated at a density of $3 \times 10^{4}$ cells $/ \mathrm{cm}^{2}$ onto poly-ornithine-coated coverslips using various multiplicities of infection (MOIs; viral particle per cell). Neurons and glials cells were plated in MEM-HS for $4 \mathrm{~h}$ before addition of the recombinant lentiviral suspension. Cell-virus contact was carried out for $1 \mathrm{~h}$ in Neurobasal-B27 glia-conditioning growth medium. Cultures were maintained in growth medium until fixation in cold methanol. We checked recombinase efficacy and cell viability by comparing LV-Cre $V e z t^{f l f l}$ to control Vezt ${ }^{+/+}$neurons either untransduced or transduced with a lentivirus-Pgk-cre (LV-Cre). We used lenti-Pgk-GFP (LV-GFP) transduction as a control of virus toxicity, which could be specifically associated with the recombinase activity from the LV-Cre. We optimized cell-virus culture conditions by comparing the number of Vezatin ${ }^{+}$ spines at 21 DIV after virus transduction, fixation, and immunocytochemistry followed by analysis under optical examination using various ratios of particle units to cells ( $\mathrm{MOI}=1,0.6$, or 0.3$)$. The use of a low ratio of transducing particle units to cells $(\mathrm{MOI}=0.3$ ) shows no evident cell death and 75-90\% transduction efficacy. Quantification of the number of neurons bearing Vezatin + spines was thus carried out at 21 DIV at an MOI of 0.3 in Vezt $t^{f / f l}$ LV-Cre versus Vezt ${ }^{+/+}$LV-Cre as control neurons in two independent experiments. Efficient detection of transduced cells was performed using a monoclonal antibody directed against the Cre recombinase (1:2000; Sigma-Aldrich). Antibodies against Vezatin were used to assess the knock-down efficiency and against MAP2 to visualize the dendritic tree. Quantification of Vezatin ${ }^{+}$spots on a similar dendritic length in knock-down (Vezt $t^{f l f l} \mathrm{LV}$-Cre) and control $\left(\mathrm{Vezt}^{+/+}\right.$ LV-Cre) neurons $(n=10)$ was carried out using MetaMorph (Molecular Devices) on at least 170 Vezatin $^{+}$spots.

Morphometric analysis of dendrites and soma. Sholl analysis on MAP2immunostained neurons from Vezt $t^{f l / f l} \mathrm{LV}$-Cre and Vezt ${ }^{+/+} \mathrm{LV}$-Cre $(n=$ 14 for each class from 2 independent experiments) was carried out using the Sholl analysis plug-in in ImageJ software (National Institutes of Health, Bethesda, MD) (objective $20 \times$ ). Pictures were binarized using the same threshold value in control and mutant to select soma and dendrites from isolated neurons. According to Sholl analysis, concentric circles were drawn at $1 \mu \mathrm{m}$ intervals around the soma center up to 150 $\mu \mathrm{m}$ (Sholl, 1953). The number of crossing dendrites was counted at each of these circles to quantify dendritic branching complexity. Soma surface was calculated using ImageJ software. Dendrite length was measured using the module Neurite Outgrowth in MetaMorph (Molecular Devices) software. The numbers of total branch ends (tips) and primary dendrites were manually counted.

Immunocytochemical and confocal analysis. Brains from 5- to 24-weekold male mice were fixed with $4 \%$ PFA by transcardial perfusion, postfixed for $2 \mathrm{~h}$ in the same fixative, rinsed in PBS, and kept in PBS-30\% sucrose at $4^{\circ} \mathrm{C}$ until use. Brains were sectioned on a cryotome at $40 \mu \mathrm{m}$, and coronal floating sections were processed for immunocytochemistry as described for cultured neurons with slight modifications: fixation with cold methanol was for $10 \mathrm{~min}$ at $-20^{\circ} \mathrm{C}$ and blocking solution was PBS/ $0.25 \%$ fish gelatin $/ 5 \%$ normal donkey serum $/ 0.3 \%$ Triton. Primary antibodies and secondary donkey antibodies were incubated at $4^{\circ} \mathrm{C}$ for $40 \mathrm{~h}$ and at RT for $3 \mathrm{~h}$, respectively, in PBS/0.125\% fish gelatin/0.3\% Triton. All sections from paired cre $-/$ cre + brains were processed under identical conditions the same day. Standard epifluorescence images were acquired on a Leica DMRD microscope using a high-resolution camera (Coolsnap HQ) driven by the MetaMorph (Molecular Devices) Image Analysis System. Confocal images were acquired using a confocal microscope Leica TCS SP 2 or SP 5 and $20 \times, 40 \times$, or $63 \times$ objective with sequential acquisition settings of $1024 \times 1024$ pixels. All confocal images from brain slices with or without the Cre recombinase were acquired from paired cre-/cre + offspring on the same day and using the same imaging conditions (Gain, Offset, Numerical Aperture, Pinhole opening).

Antibodies for immunostaining. Primary antibodies and dilution used were the following: affinity-purified rabbit (L1121) polyclonal antibodies directed against the mouse Vezatin His-tagged C-terminal region (aa $350-617)$ was raised in 2006 in collaboration with Professor Christine Petit (Pasteur Institute, Collège de France, INSERM UMRS587, Paris, France). Its specificity was previously demonstrated by antigenic preadsorption using Western blotting of brain and testis extracts and immunocytochemistry on PFA/post-methanol fixed testis cryosections (Hyenne et al., 2007a, 2007b) and further used by Balhoul et al. (2009). Dilutions used here were 1:3000 for neurons in culture and 1:10000 for floating sections. Rabbit antibody anti-GluA2 was used at 1:200 (Alomone Labs). Mouse monoclonal antibodies were as follows: anti-N-cadherin (clone 32), 1:100 (BD Biosciences); anti-GluA2 (clone 6C4), 1:400 (Millipore); anti-Syb2 (clone 69.1), 1:5000 (Synaptic Systems); anti-PSD95 (MA1046), 1:250 (Affinity BioReagents); anti-GAD67 (clone 1G10.2), 1:250 (Millipore); anti-GAD65 (MAB351), 1:500 (Millipore), anti-Bassoon (VAMPS003), 1:500 (Stressgen), and anti-GFAP (clone GA5), 1:400 (Sigma-Aldrich). Guinea pig polyclonal anti-synapsin 1/2 antibodies (106004) were from Synaptic Systems and used at 1:1000. Chicken antiMAP2 antibodies (ab5392) used at 1:10000 were from Abcam. Phalloidin staining and secondary antibodies were the following: Alexa Fluor 568 phalloidin (1:250), Alexa Fluor 488 donkey-anti-mouse IgG (1:500) (A21202), and donkey-anti-rabbit IgG (1:500) (A21206), purchased from Applied Biosystems. Cy3-conjugated AffiniPure donkey-anti-rabbit IgG (711-165-152) and donkey-anti-mouse ( $\left.\mathrm{F}\left(\mathrm{ab}^{\prime}\right) 2\right)(711-166-151)$ were from Jackson ImmunoResearch Laboratories and used at 1:400. Cy5-conjugated AffiniPure donkey-anti-chicken IgG (703-175-155), -anti-mouse IgG (715175-151), and -anti-guinea pig IgG (706-175-148) were purchased from Jackson ImmunoResearch Laboratories and used at 1:250. Normal donkey serum was from Jackson ImmunoResearch Laboratories.

Quantification of postsynaptic GluA2 and Vezatin clusters. Floating slices from $D 6 c K O$ and control brains (4-week-old males; $n=2$ ) were stained with rabbit antibody against GluA2 (extracellular). Vezatin was used to control extinction. We imaged the CA1 field corresponding to the stratum radiatum (SR) region by using a laser scanning confocal microscope (objective $40 \times$, zoom 2.67). A $z$-stack for each of 10 subregions $(14.35 \times 14.35 \mu \mathrm{m})$ was collected for a total depth of $12 \mu \mathrm{m}(12 \times 1 \mu \mathrm{m}$ 
optical sections). Numerical output and quantification of the analysis of GluA2 cluster number was carried out using MetaMorph (Molecular Devices). To measure Vezatin expression in $\mathrm{CKO}$, we stained paired sections from control and cKO littermate males (2- to 4-month-old males, 3 pairs of CamKIIcre-/cre +, and 2 pairs of $D 6 \mathrm{cre}-/ \mathrm{cre}+$ ) under identical conditions. Images of CA1 SR were acquired by confocal microscopy (Leica SP5 confocal microscope, $40 \times$ objective) under identical parameters in cre + and cre - male offspring on the same day. We quantified the intensity of Vezatin staining in MAP2-positive dendritic branches, with a dedicated script using the Integrated Morphometric Analysis module of MetaMorph (Molecular Devices). Efficiency of immunostaining was controlled by calculating the ratio of Vezatin intensity to that of MAP2.

Golgi-Cox impregnation and spine quantification in vivo. Anesthetized mice were perfused for $10 \mathrm{~min}$ with $0.9 \% \mathrm{NaCl}$ solution. Brains of 8 -week-old CamKIIcKO and control male mice ( $n=3$ for each genotype) were immersed for $2 \mathrm{~d}$ in Golgi-Cox solution ( $1 \%$ potassium dichromate, $1 \%$ mercuric chloride and $0.8 \%$ potassium chromate) (Glaser and Van der Loos, 1981). Subsequently, brains were preserved in $30 \%$ sucrose/Milli-Q (Millipore) water solution, regularly changing the solution for 1 week. Serial coronal sections of the hippocampus $(200 \mu \mathrm{m})$ were cut using a sliding vibratome and preserved in $6 \%$ sucrose/Milli-Q (Millipore) water solution (Gibb and Kolb, 1998). Processing of the sections involved development in ammonium hydroxide (30\%), fixation in photographic fixative (Eastman Kodak), dehydration, and clearing in xylene. Sections were mounted onto gelatinized glass slides and coverslipped with poly butyl methacrylate-co-methyl methacrylate (Eukitt; Kindler $\mathrm{GmbH}$ ). Image acquisition of dendritic fragments was conducted by using a combined hardware-software system (Axiovision 4.7; Zeiss) controlling the $x-y-z$ axis of the microscope (AxioVert 200M; Zeiss) and the microscope-mounted video camera (AxioCam MRm; Zeiss) using a $63 \times / 1.32$ objective. The size of the pixels was $100 \times 100 \times$ $130 \mu \mathrm{m}$. Dendrites and spines were visualized using 3D images with the help of Imaris software 7.0 (Bitplane Scientific Software), inverted colors (LUTINV), and built-in tools (see below). Dendritic segments were analyzed in blind scoring conditions. Dendrites were tracked with the FilamentTracer module (Bitplane Scientific Software), which by means of an autopath method semiautomatically labels dendrites with a blue cylinder of $0.25 \mu \mathrm{m}$ diameter according to the range values of the diameter of distal dendrites $(0.25-1 \mu \mathrm{m})$ in SR (Fiala et al., 2008). On the automatically labeled dendrites, Golgi stains were manually indicated under interactive $3 \mathrm{D}$ visualization as red spots (diameter of $0.3 \mu \mathrm{m}$ ) using the built-in Spot detection module. Staining in each dendritic segment was thresholded manually such that all visually discernible protuberances in three dimensions were identified according to the range values of the length of spines in CA1 $(0.3-2 \mu \mathrm{m})$ (Harris and Stevens, 1989). Stained spots distributed away from a segment of dendrite ( $3 \mu \mathrm{m}$ away) were excluded from the analysis. Marked spots were then categorized into two types. Spots lying within or touching the edge of the dendritic blue cylinder (i.e., without a clear neck) were classified as stubby spines. Spots away from the blue cylinder but within a margin of $3 \mu \mathrm{m}$ from the edge of the filament were classified as spines.

Behavioral analyses. Cohorts of 3- to 4-month-old CamKIIcKO $\left(\right.$ Vezt $t^{l l f l}::$ CamKIIcre +$)$ and control $\left(\right.$ Vezt $\left.t^{f l / f l}\right)$ male mice were used. Body weights between two groups of 10-12 mice of each genotype at 4 months of age were not significantly different ( $p=0.089$, Student's $t$ test). Preyer reflex to a flick backward of the pinna to a sharp, calibrated, intense sound burst (Steel and Hardisty, 1996) showed no major auditory defect. One cohort was successively tested in open field, elevated plus maze, and passive avoidance. Two cohorts were tested in Morris Water Maze (MWM) in identical conditions, one of which was also tested in contextual and cued fear conditioning. We verified the decreased Vezatin immunoreactivity by immunochemistry in the hippocampus of CamKIIcKO compared to control littermates from the two cohorts used in MWM at 6 months of age. Control $(n=12)$ and $(n=10)$ CamKIIcKO male mice were explored in open field, elevated plus maze, and passive avoidance by Key-Obs SA (Parc Technologique).

Open field. Total activity and anxiety-related responses were assessed by comparing the time, the distance (at the periphery or in the center), and the number of rearing in a clear square arena $(45 \times 45 \times 40 \mathrm{~cm})$ for
$30 \mathrm{~min}$. Assessment takes place over $2 \mathrm{~d}$ to allow habituation to the environment to be evaluated (two sessions). Exploration behavior was video tracked and automatically monitored every 5 min for six consecutive intervals.

Elevated plus maze. The apparatus consisted of two open arms and two closed arms $(26 \times 6 \mathrm{~cm})$ having $12 \mathrm{~cm}$ tall sides. The entire apparatus was mounted $50 \mathrm{~cm}$ above the surface of the floor. This test allows measurement of the percentage of time spent and the number of entries in open arms (i.e., to evaluate the conflict between the natural tendency to explore a novel environment vs the tendency to avoid the aversive properties of an open, narrow, elevated surface). In this test, greater amount of time spent as well as higher number of entries in the open arms indicate a decrease in state anxiety. Each test was begun by placing the mouse in the center of the maze, which was left free to explore the maze for $5 \mathrm{~min}$. Mice were scored for the amount of time spent and the number of entries into the open and closed arms, determined by placement of all four paws into that area. Exploration behavior was video tracked and automatically monitored during the 5 min test. Entries into the closed arms were assessed as a measure of activity. Entries and time spent into the open arms were assessed as a measure of anxiety.

Passive avoidance. Mice were tested using a two compartment $(25 \times$ $9 \times 45 \mathrm{~cm}$ ) box with a lighted and a dark compartment with a footshock grid on the floor. This test allows measurement of the latency of a mouse to enter a dark compartment of the double chamber that it prefers but that is associated with a footshock (i.e., to measure the fear response after an unpleasant shock). Wild-type mice are slow to enter. On the training day (acquisition), each animal was placed in the illuminated box and the interchamber door was closed. After a $60 \mathrm{~s}$ habituation, the door was opened. When the mouse crossed to the darkened side, the door was closed and a $0.4 \mathrm{~mA}$ for $0.5 \mathrm{~s}$ footshock was delivered. For the test retention trial, animals were brought back to the room after the appropriate interval and run through the same protocol but without footshock. For both acquisition and retention trials, the latency to cross to the dark was measured using photocells in the boxes.

MWM. Control $(n=19)$ and CamKIIcKO $(\mathrm{n}=19)$ male mice were tested according to the procedure of Law et al. (2003). All trials were video recorded by computerized monitoring software (Viewpoint). The mean of two consecutive trials was used for graphical representation and statistical analysis. Control and cKO mice were considered in our analysis after exclusion of those mice that performed badly with prolonged floating behavior (three controls and six CamKIIcKO mice). Mice were trained in a $136 \mathrm{~cm}$ diameter circular pool (water at $20 \pm 1^{\circ} \mathrm{C}$, made opaque by a nontoxic white paint) with a $13 \times 13 \mathrm{~cm}$ platform placed 0.5 $\mathrm{cm}$ below the water surface. The maze was virtually divided into four arbitrary, equally spaced quadrants delineated by the cardinal points north $(\mathrm{N})$, east $(\mathrm{E})$, south $(\mathrm{S})$, and west $(\mathrm{W})$. The pool was located in a large room $(5 \times 4 \mathrm{~m})$ with extra maze cues including brightly colored geometric and high-contrast images (squares, stars, posters) mounted on the walls and with the ceiling illuminated through upward diffuse lighting. Mice were first trained with a cued platform (d $1-2 ; 4$ trials per $\mathrm{d}$; intertrial interval of $1 \mathrm{~h}$ ) so that they could associate the platform with escape from the pool. The pool was surrounded by a white curtain to occlude the sight of extra maze cues. The platform was cued by a $15 \mathrm{~cm}$ high dark cylinder placed on it and located in different locations across trials. Mice were started from four symmetrical positions in a pseudorandomized manner for two sessions, one session a day. Each session consisted of four trials placing the mouse in the pool, facing the wall of the tank. The mouse was allowed $90 \mathrm{~s}$ to search for and climb onto the submerged platform. If the animal failed to locate the platform within this delay, it was subsequently placed on the platform by the experimenter. In all cases, the mouse was left on the platform for $15 \mathrm{~s}$. Distance traveled and latency (total and in each quadrant) before finding the platform were collected for each mouse. For the spatial learning session, all mice were trained over $5 \mathrm{~d}$ ( $\mathrm{d} \mathrm{3-7;} 6$ trials per d; intertrial interval of $50 \mathrm{~min}$ ) in the presence of extra maze cues. Traveled distance and latency (total and in each quadrant) were collected for each mouse. For the third trial on $\mathrm{d} 7$, the platform was removed, and mice were allowed to swim for $60 \mathrm{~s}$ (transfer trial, first probe test). Additional parameters were noted: 
latency before reaching the target quadrant (NE), latency before finding the platform position, time spent in the target quadrant, and number of times crossing the previous place of the platform. For relearning $(\mathrm{d} 8-11)$, the hidden platform was placed at the center of quadrant SW. All mice were trained over $4 \mathrm{~d}(\mathrm{~d} 8-11 ; 6$ trials per $\mathrm{d}$; intertrial interval of $50 \mathrm{~min}$ ) in the presence of extra maze cues. Distance traveled and latency (total and in each quadrant) were collected for each mouse. Three days after the final relearning trial, all animals were tested for transfer trial (second probe test). The same parameters were recorded as in the first probe test. To prevent hypothermia, the animals were gently dried with a cotton towel between and after the trials.

Contextual and cued fear conditioning. Control $(n=9)$ and CamKIIcKO $(n=9)$ male mice were tested as previously described by Obiang et al. (2011) using a computer-controlled fear conditioning system (BIOSEB). Fear conditioning is a form of learning associating an adversive stimulus (electrical shock) with a complex environment (a context) or to an elemental cue (a tone). It results in a conditioned response (fear) easily measurable as freezing in rodents. After a habituation phase ( 2 sessions of $5 \mathrm{~min}$; $24 \mathrm{~h}$ intertrial interval), the conditioning phase was performed on the third day. Mice were then placed back in the conditioning chamber and left free to explore for 2 min. Two $30 \mathrm{~s} 2000 \mathrm{~Hz}$ pure tone (conditioned stimuli) separated by $2 \mathrm{~min}$ were then delivered, each coterminating with a $2 \mathrm{~s}, 0.4 \mathrm{~mA}$ footshock (unconditioned stimulus). Thirty seconds after the final shock, mice were returned to their home cages. Contextual fear memory was assessed $24 \mathrm{~h}$ after conditioning by returning the mice to the conditioning chamber and measuring freezing behavior during a 4 min retention test. Cued testing with altered context (odor, color, light) was performed $48 \mathrm{~h}$ after conditioning, after a period of $120 \mathrm{~s}$ free exploration followed by the delivery of a $2000 \mathrm{~Hz}$ tone during a $120 \mathrm{~s}$ retention period. The measurement of fear was performed by considering the freezing time, defined as immobility (i.e., the absence of all movements with the exception of those related to respiration) for at least a period of $1 \mathrm{~s}$.

Electrophysiology. Hippocampal slices were prepared from 18- to 24d-old D6cKO or control male mice as described previously (Scheuber et al., 2006). Slices were maintained at $31^{\circ} \mathrm{C}$ and superfused with artificial CSF containing the following (in mM): $124 \mathrm{NaCl} ; 26.2 \mathrm{NaHCO}_{3} ; 11$ glucose; $3 \mathrm{CaCl}_{2} ; 2.5 \mathrm{KCl} ; 2 \mathrm{MgCl}_{2}$. Whole-cell recordings were made from CA1 pyramidal cells using borosilicate glass microelectrodes $(2-5$ $\mathrm{M} \Omega$ ) filled with an intracellular solution containing the following (in mM): $100 \mathrm{CsMeSO}_{4} ; 20 \mathrm{CsCl} ; 10$ HEPES; 10 EGTA; 4 MgATP; 0.4 $\mathrm{Na}_{3}$ GTP, pH 7.4 (290 mOsm). mEPSCs were recorded while holding cells at $-70 \mathrm{mV}$ in the presence of $1 \mu \mathrm{M}$ TTX and $20 \mu \mathrm{M}$ bicuculline methochloride. mEPSCs were detected and analyzed semiautomatically using DETECTiVENT (LabView) software (Ankri et al., 1994). Evoked EPSCs were recorded upon extracellular stimulation of Schaffer collaterals in the presence of bicuculline methochloride $(20 \mu \mathrm{M})$ after a cut was made between CA3 and CA1 areas. AMPAR- and NMDAR-mediated currents were measured from 50 consecutive episodes recorded at -60 and $+40 \mathrm{mV}$, respectively. To compare AMPA/NMDA currents ratios, stimulation intensities were adjusted to induce EPSCs of similar amplitudes when recorded at $-60 \mathrm{mV}$. AMPAR-mediated EPSC amplitudes were measured within a $2 \mathrm{~ms}$ window centered on the peak of the current. For NMDAR-mediated EPSCs, amplitude was measured within a $20 \mathrm{~ms}$ window positioned $30 \mathrm{~ms}$ after stimulation artifact. For display, stimulation artifacts were digitally clipped. Values are expressed as mean \pm SEM. Statistical significance was estimated using Mann-Whitney $U$ tests performed under SigmaStat (SPSS).

Statistics. All data presented in the text are mean \pm SEM. Behavioral data were analyzed by ANOVA with repeated measurements (having genotype as the between-groups factor), followed by post hoc analysis (Newman-Keuls) when appropriate. The Mann-Whitney $U$ test was used to compare quantitative variables between groups. Statistical significance in the morphometric MAP2 analysis was established at $p<0.05$ and $p<0.001$. Statistical analysis and graph representations were carried out using GraphPad Prism programs.

\section{Results}

Vezatin is enriched in spines in hippocampal neurons in vitro and in vivo

We investigated Vezatin distribution using cultured hippocampal neurons. Figure $1 A$ shows that Vezatin was mainly expressed in the soma and growth cones at 5 DIV and later (14-21 DIV) concentrated in the somato-dendritic compartment as small puncta suggestive of localization in spines. We tested whether Vezatin was also expressed in presynaptic elements by comparing immunostaining for Vezatin and Synaptobrevin2 (Syb2), a vesicular presynaptic marker. Vezatin does not colocalize with Syb2 either in young or in developing neurons (Fig. $1 B$ ). N-cadherin distributes at both sides of the synapses and is required for synapse formation/plasticity (Arikkath and Reichardt, 2008). In mature synapses, Vezatin and N-cadherin partially colocalized in spines, reinforcing the notion that Vezatin is only on one side of the synapse in contrast to $\mathrm{N}$-cadherin (Fig. 1C/C1). In addition, Vezatin was expressed in some GABAergic interneurons, as revealed by coimmunostaining with GAD67 (Fig. $1 D$, left). However, no colocalization of Vezatin with GAD65 enriched in presynaptic terminals of interneurons was observed (Fig. 1D, right). Together these results indicate that Vezatin is enriched in the postsynaptic compartment of both excitatory and inhibitory neurons.

Since Vezatin is indirectly associated with actin (KusselAndermann et al., 2000; Bahloul et al., 2009), we compared Vezatin and F-actin distributions. In immature neurons, Vezatin immunoreactivity was found in growth cones at the tips of growing neurites displaying an arc-shaped F-actin + distribution (Fig. $1 E$ ) (see also a fixed neuron, in top inset). This region corresponds to a specialized zone of contractile F-actin containing Myo IIB (transition zone) (Medeiros et al., 2006). In developing neurons, Vezatin colocalized with phalloidin (labeling F-actin) in dendrites and spines (Fig. $1 F$ ). We checked the specificity of the affinity-purified anti-Vezatin polyclonal antibodies in brain by quantifying Vezatin expression level in hippocampi extracts from adult control Vezt $t^{f l / f l}$ and heterozygous Vezt $t^{f l n u l l}$ mice carrying one floxed allele together with one null allele originating from zygotic Vezatin invalidation (see Materials and Methods). As expected, quantification of the highest band that corresponded to one of the two major Vezatin isoforms (2.4 kb isoform, AY75561) showed a $56.5 \pm 7 \%$ reduction in heterozygous hippocampi compared to control extracts ( $n=3$ experiments). The band at 71 $\mathrm{kDa}$ corresponding to the second major Vezatin isoform $(1.9 \mathrm{~kb}$ isoform, AY753562) was also reduced by $\sim 50 \%$ in the heterozygous hippocampi. Lower bands likely corresponded to splice variants and/or proteolytic fragments, which at this point have not been characterized in great detail (Fig. 1G). This result confirmed our previous antibody specificity controls (Hyenne et al., 2007a, 2007b).

We next examined Vezatin distribution relative to the commonly defined subtypes of protrusions: filopodia, stubby, thin, and mushroom spines (Parnass et al., 2000). Vezatin was expressed in filopodia given as transient precursor spine structures observed only during early normal development (Fig. 2A, left) and in thin, stubby, and mushroom spines (Fig. $2 A$, left and right). To investigate Vezatin distribution within spine in mature neurons, we colabeled cultured hippocampal neurons at 21 DIV with Vezatin and PSD95, a marker of the postsynaptic density (PSD). PSD95 is a neuronal PDZ protein that associates with receptors/cytoskeletal elements at functional synapses (Sheng and Hoogenraad, 2007). At high magnification, Vezatin clearly 
colocalized with PSD95 in spine heads in mushroom and stubby spines (Fig. 2B). However, Vezatin codistributed with PSD95 only at PSD edges, encircling the PSD95 region (Fig. 2C). We quantified the overlap of Vezatin + and PSD95+ staining by confocal microscopy in 17 individual spines. About half of the Vezatin+ pixels (54.7\%) colocalized with the PSD95+ pixels while most of the PSD95+ pixels $(85.9 \%)$ colocalized with Vezatin + pixels in 21 DIV neurons. These proportions were not significantly different at $14 \mathrm{DIV}$ versus 21 DIV $(p=0.2415$ and $p=0.2346$, MannWhitney $U$ test). The large proportion of PSD95+ pixels that colocalize with Vezatin + pixels reinforces the conclusion based on imaging data that Vezatin codistributes with PSD95 expression territory, PSD95 expression alone being restricted only at the extreme tip of the spine head. We also observed that Vezatin distributed beyond the spine head toward the neck (Fig. 2B,C). Consistent with a distribution of Vezatin at synaptic contacts, crude subcellular fractions of hippocampi were evaluated by immunoblotting. Like PSD95, GluA2, and GluN1 (two glutamate synaptic receptors), Vezatin was abundant in the crude synaptosomal membrane fraction (Fig. 2D). Vezatin also partitioned in the cytosolic fraction, which most probably corresponded to the cytoplasmic immunostaining observed in many cells (Kussel-Andermann et al., 2000; Hyenne et al., 2005; Bahloul et al., 2009). In mature neurons Vezatin and phalloidin colocalized with PSD95, adopting a cup shape distribution at the base of the spine head with a tip staining specific to PSD95 as described above (Fig. 2E). These results thus show that Vezatin is present in developing/ mature spines during neuronal differentiation over time, where it localizes F-actin.

To examine Vezatin localization in vivo, we immunostained adult brain sections. Vezatin was mainly expressed in the hippocampus, cortex, and medial habenula $(\mathrm{MHb})$. The striatum, cerebral peduncle, amygdala, and thalamus showed moderate staining (Fig. 2F). Within the cytoarchitectonic laminar organization of the hippocampus, Vezatin was mainly observed in soma from principal neurons of CA1/CA3 and DG fields at low-power resolution (Fig. 2G). The hilar region adjacent to dentate granule cells was devoid of Vezatin staining, except in inhibitory neurons and/or astrocytes, suggesting that Vezatin was poorly expressed in axons if any (Fig. 2G). At higher resolution, Vezatin is expressed diffusely within the cytoplasm (Fig. 2G1) and sometimes in the nucleus of pyramidal neurons (left). The Vezatin localization within the cytoplasm is in agreement with previous results showing diffuse immunostaining in the cell body of nonconfluent epithelial cells (Kussel-Andermann

E
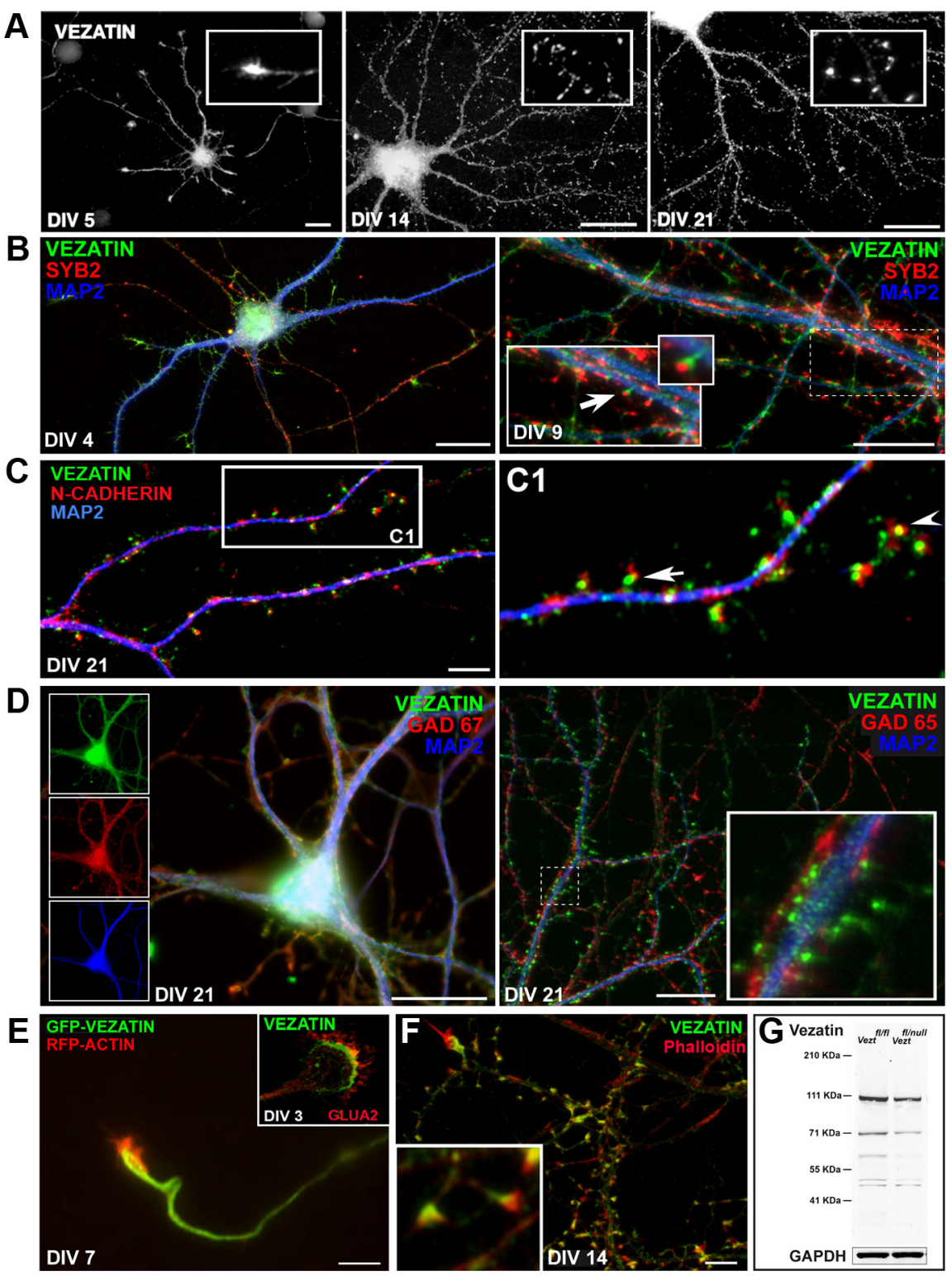

Figure 1. Vezatin concentrates in spines during neuronal differentiation. $\boldsymbol{A}$, Developmental distribution of Vezatin showing its concentration in growth cone and dendrites early during neuronal differentiation (inset, left) and in dendrites and spines in maturing and mature neurons (insets, middle and right). $\boldsymbol{B}$, At the early stage of neuronal differentiation (left) and later, at the onset of dendritic spine development, Vezatin does not colocalize with presynaptic Syb2 (right, arrow). C, Postsynaptic Vezatin is partially codistributed with transsynaptic N-cadherin. (1, The arrowhead and the arrow are pointing to a spine where Vezatin is (yellow) and is not (red aside green) clearly overlapping the $\mathrm{N}$-cadherin signal, respectively. $\boldsymbol{D}$, Vezatin is expressed in the soma of interneurons stained with GAD67 (left). Vezatin does not codistribute with GAD65 at the axonal compartment (right). MAP2 is used to label dendrite. $\boldsymbol{E}$, Snapshot of a time-lapse image of a living cortical neuron transfected with Vezatin-GFP and RFP-actin showing codistribution in a hemicircumferential ring in a growth cone. Top, inset, DIV 3, fixed cultured hippocampal neuron immunostained for Vezatin and GluA2, a glutamate receptor, showing that Vezatin expression is restricted to an arc-shaped region of the growth cone. $\boldsymbol{F}$, Vezatin colocalizes with phalloidin (F-actin) in dendrites and in developing spines at early synaptic sites (DIV 14). Scale bars, $10 \mu \mathrm{m}$. G, Protein expression in mouse hippocampi extracts. The anti-Vezatin antibody recognized the two major Vezatin isoforms (see text) with band intensities approximately $2 \times$ weaker in the heterozygous floxed/null ( $\mathrm{fl} /$ null) compared to the floxed/floxed (fl/fI) extract. Other few alternatively spliced variants are expressed in minor quantities. Loading control, GAPDH.

et al., 2000; Bahloul et al., 2009). Nuclear expression can be found in blastula in vivo (Hyenne et al., 2005) or in cultured cells in vitro (Figs. $3 A$, neurons, $4 B 1$, astrocytes). Furthermore, Vezatin is expressed in dendrites (Fig. 2G1, left) and spines (Fig. 2G1, middle), partially codistributing with $\mathrm{N}$-cadherin in spines in the CA1 field. In the CA3 region, Vezatin is also expressed in soma and thorny excrescences, the postsynaptic compartment synapsing with the large boutons of mossy fiber axons from dentate 

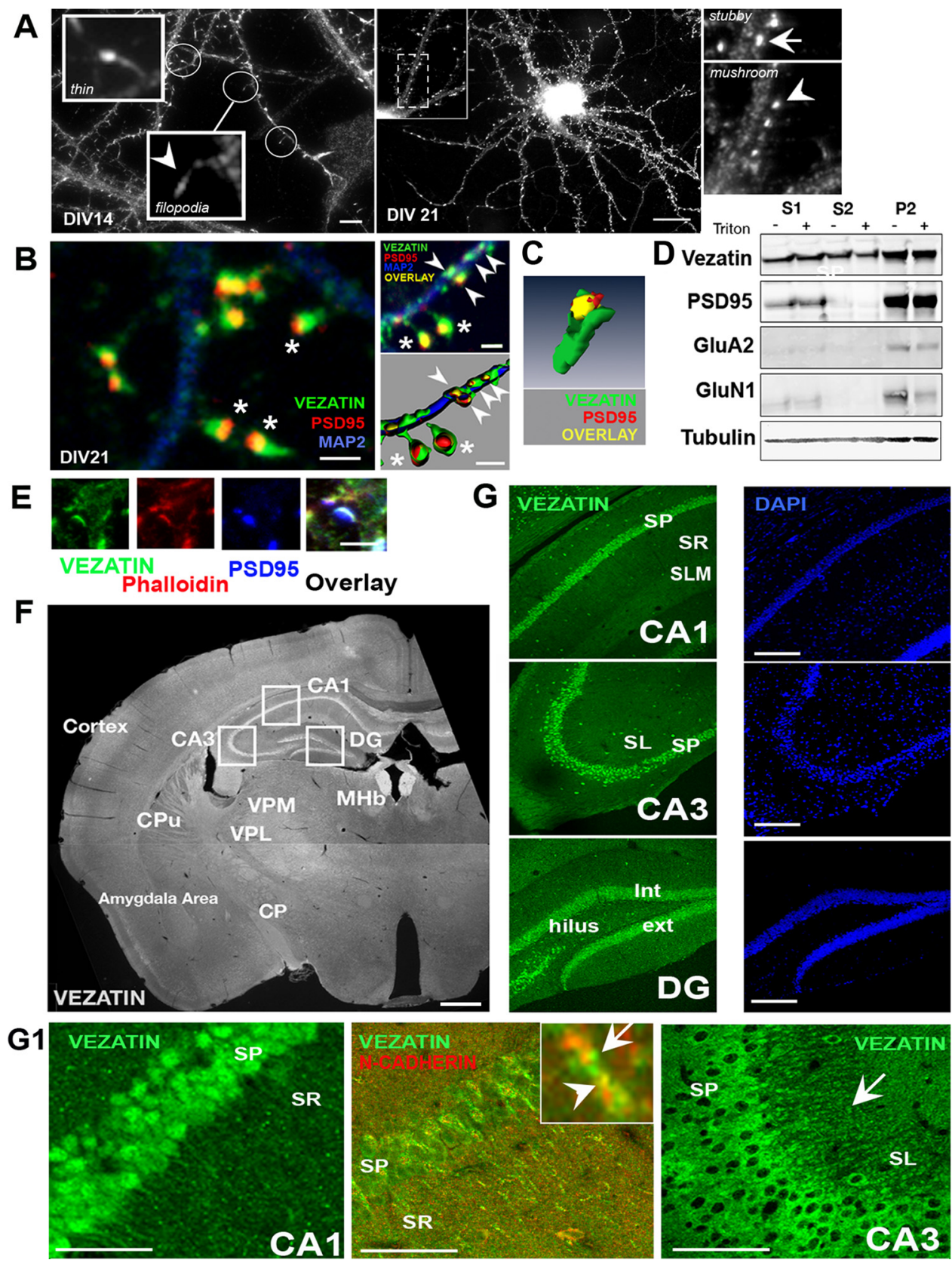

Figure 2. Subcellular spine Vezatin expression in vitro $(\boldsymbol{A}-\boldsymbol{E})$ and in vivo $(\boldsymbol{F}-\boldsymbol{G}) . \boldsymbol{A}$, Vezatin labels all stages of spine differentiation. Insets, Higher magnifications of a filopodium (arrowhead) and a thin spine (left). A stubby (arrow) and a mushroom-like (arrowhead) are shown on the right (top and bottom insets). B, Confocal images showing that Vezatin (green) and PSD95 (red) colocalize (yellow) at spine heads. Asterisks and arrowheads point to representative mushroom and stubby spines, respectively. Bottom right, Reconstructed view using the Imaris software package. C, Vezatin extends beyond the PSD95/PSD margin toward the neck of the spine: snapshot of a confocal spine 3D reconstruction (21 DIV). Overlay (yellow) between Vezatin (green) and PSD95 (red) staining. Both markers colocalize at the tip of the spine head, but PSD95 is more central than Vezatin. D, Vezatin is in the synaptosomal membrane fraction as PSD95 or GluA2 and GluN1 (P2). Vezatin is also in the cytosolic fraction (S2). Crude protein extract was obtained by clearing thelysate by gentle centrifugation (S1) followed by high-speed centrifugation (S2 and P2).E, High-magnification images of a 21 DIV spine whose cup-shaped base is colabeled (overlay) with Vezatin (green), phalloidin (red), and PSD95 (blue). Scale bars: $\boldsymbol{A}, 10 \mu \mathrm{m} ; \boldsymbol{B}$ (left), $\boldsymbol{E}, 2 \mu \mathrm{m} ; \boldsymbol{B}$ (right), $1 \mu \mathrm{m} . \boldsymbol{F}$, Vezatin is expressed in hippocampus, cortex, and MHb. Other regions including striatum [caudate putamen (CPu) and cerebral peduncle (CP)], amygdala, and thalamus [ventral postero-medial thalamic nucleus (VPM) and ventral postero-lateral thalamic nucleus (VPL)] expressed Vezatin at a low level (objective $1.6 \times$ ). Scale bar: $1000 \mu \mathrm{m}$. G, Confocal microscopy at low-power resolution indicates that Vezatin is expressed in soma (objective 20X) [SP in CA1/CA3 and interior/exterior (int/ext) in DG]. No staining is observed in the hilus (DG), except in cells attributed to astrocytes and/or inhibitory neurons. DAPI staining is used to label nuclei. G1, Higher-power resolution (objective 40 X,z0om 2.67). Left, Vezatin is in dendrites (SR in CA1). Middle, Vezatin is in spines partially codistributing with the transsynaptic N-cadherin marker (CA1 SR region). Inset, Arrowhead and arrow are pointing to spines where postsynaptic Vezatin (green) is (yellow) and is not (green aside red) overlapping N-cadherin (red). Right, Vezatin is in postsynaptic thorny excrescences (in SL in CA3) that mark the location of mossy fiber synaptic terminations (arrow). Scale bars: $\mathbf{G}, 400 \mu \mathrm{m} ; \mathbf{G 1}, 100 \mu \mathrm{m}$. SP, stratum pyramidale; SL, stratum lacunosum.

granule cells (Fig. $2 G 1$ right). Together with our in vitro data, the expression pattern of Vezatin in vivo indicates that Vezatin is expressed in the somato-dendritic compartment and enriched in spines.
Alterations in dendritic branching in Vezatin knock-down neurons in vitro

To get a first insight on the potential role of Vezatin in neuronal morphogenesis, the effect of Vezatin knock-down was tested in 
A ver
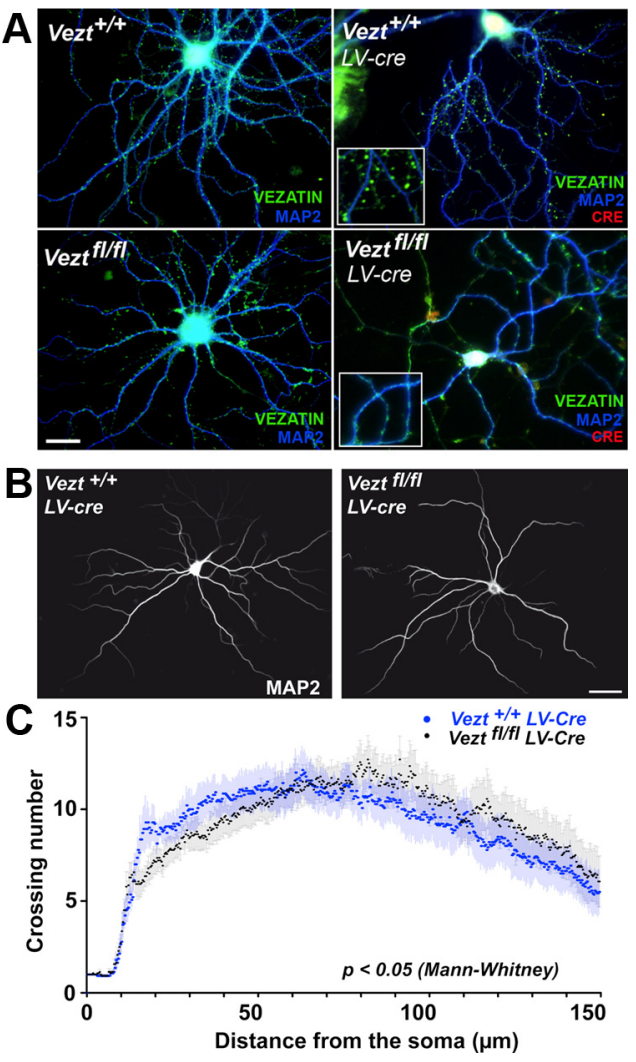

Figure 3. Vezatin knock-down induces less dendritic branching complexity in vitro. $\boldsymbol{A}$, Vezatin expression is efficiently knocked down: absence/poor Vezatin staining is observed in $88.2 \%$ LV-cre Vezt ${ }^{f / f l}$ neurons (30/34) (see a representative neuron with stubby-like signals along dendrites, right bottom). In contrast, $83.8 \%$ LV-cre Vezt $^{+/+}$neurons (31/37) are decorated with spine-like Vezatin + spots (right top), a percentage similar to that obtained in Vezt ${ }^{+/+}$ $(91.4 \%, 32 / 35)$ or Vezt $t^{f l f l}(94.1 \%, 32 / 34)$ neurons (left top/bottom). MAP2 and Cre antibodies visualize dendrites and transduced neurons, respectively (right). Scale bar, $10 \mu \mathrm{m}$. $\boldsymbol{B}$, Vezatin knock-down impairs dendritic branching but not length. Representative control (left) and knock-down (right) neuron fixed at 21 DIV and immunostained with anti-MAP2 antibodies. Scale bar, $50 \mu \mathrm{m}$. $C$, The number of crossing dendrites is overall significantly altered in knockdown (dark circles) compared to control (blue circles) neurons $(p<0.05)$. The decrease is highly significant in the proximal region $(p<0.001)$.

vitro on 21 DIV hippocampal-dissociated neurons from Vezt $t^{f l f l}$ brains after transduction with a Cre-expressing lentiviral vector (LV-Cre) at d 0 . We examined neurons by immunofluorescence analysis using MAP2 as a dendritic marker and anti-Vezatin and anti-Cre antibodies to follow the knock-down and transduction efficacy, respectively. At least 30 neurons were examined in two independent experiments. Following transduction with a low virus-cell ratio, the vast majority of transduced $V e z t^{+/+}$control neurons (31/37) were decorated with Vezatin + bulbous spots resembling spines (Fig. 3A, right top) as in Vezt $t^{+/+}$and Vezt $t^{f l / f l}$ neurons (Fig. 3A, left). By contrast, the vast majority of transduced Vezt $t^{f l f l}$ neurons (30/34) showed no or poor Vezatin immunoreactive staining. This indicated that Vezatin is efficiently knocked down after lentiviral-induced Cre recombination (Fig. $3 A$, right bottom).

Among 30 analyzed knock-down neurons, absence or near absence of staining occurred in 13 and 7 neurons, respectively. The remaining 10 knock-down neurons showed mostly flattened stubby-like signals (Fig. $3 A$, right bottom). We categorized the staining in these 10 knock-down ( Vezt ${ }^{f l / f l} \mathrm{LV}$-cre) and 10 control $\left(\mathrm{Vezt}^{+/+} \mathrm{LV}\right.$-cre) neurons into two categories: stubby-like (flattened, i.e., without a neck) and spine-like (bulbous, i.e., with a neck) staining. We then quantified their numbers on a similar dendritic length $(n=16-20$ dendritic segments, two experiments). Knock-down neurons displayed $81.1 \%$ of stubby-like structures (138/170). By contrast, control neurons showed $80.8 \%$ of spine-like structures $(249 / 308)(p<0.0001$, Mann-Whitney $U$ test).

To examine whether Vezatin knock-down has an effect on dendritic extension and complexity, we characterized transduced knock-down and $+/+$ neurons fixed and immunostained with MAP2 at 21 DIV ( $n=14$ in each type in two independent experiments). We quantified dendritic tree complexity by counting dendrite intersections with concentric rings drawn around the cell body at $1 \mu \mathrm{m}$ intervals at a distance of $150 \mu \mathrm{m}$ from the soma (Sholl, 1953; Kawabe et al., 2010). Knock-down neurons showed altered dendrite complexity compared to control neurons $(p<$ 0.05 ) (Fig. 3C). The size of the soma, the dendritic length, the number of primary dendrites, and the number of total branch ends were not significantly different between knock-down and control neurons $(p=0.3953, p=0.8664, p=0.4364$, and $p=$ 0.8823 , respectively). These results suggest that Vezatin is required for dendritic branching (altered number of crossings in knock-down vs control neurons) but not for neurite extension.

\section{Increased proportion of stubby spines versus spines in CamKIIcKO hippocampal neurons in vivo}

To examine further the role of Vezatin in spine morphology, we generated Vezatin conditional KO mice depleted of Vezatin only in forebrain areas after birth by crossing Vezt flfl with CamKIIcre transgenic mice (Casanova et al., 2001) (named CamKIIcKO). The timing of expression of the CamKII $\alpha$ gene (from P15-P21 onward) allowed us to examine spine morphology without possible confounds due to early developmental effects. The efficiency of Vezatin invalidation was checked at the mRNA level as in Balhoul et al. (2009) (Fig. 4A). Because Vezatin had a broad cell expression pattern in the brain, including excitatory neurons but also glial cells, GABA neurons (Fig. 4B,C), and endothelial cells, we relied on immunocytochemistry in brain sections to validate the extinction at the protein level. In hippocampus, no cellular layer disorganization was noted both in the dorsal and in the ventral poles. Vezatin immunoreactivity was comparable in the $\mathrm{MHb}$, and no difference was observed in the amygdala areas between the two genotypes. Vezatin staining was clearly decreased but not abolished in cre-expressing hippocampi versus controls in the soma of neuronal layers (Fig. 4D, CA1). Alternatively, we suspect that the remaining staining in $\mathrm{CKO}$ neuronal layers could correspond to interneuron soma. The expression decrease was particularly evident in MAP2-positive dendritic branches (Fig. $4 D$, SR). Quantification of the staining showed a decrease of $61 \pm$ $0.89 \%$ of Vezatin expression in cKO versus control [Vezt/MAP2 ratio $=0.1759 \mathrm{au}(n=576$ dendritic branches in $\mathrm{cKO})$ vs 0.4564 au $(n=627$ branches in control); $p<0.001$, Mann-Whitney $U$ test; $n=3$ pairs of cre-/cre + mice]. These results indicate that Vezatin expression is significantly reduced in adult hippocampal CamKIIcKO neurons.

To address whether ablation of Vezatin in vivo has an effect on spine morphology, whole brains from CamKIIcKO and control males ( $n=3$ males for each genotype from two litters born the same day) were stained using the Golgi-Cox impregnation protocol. We focused on the apical distal $(>150 \mu \mathrm{m}$ from cell soma) dendritic region in the CA1 field that receives input from the CA3 projections. No difference in staining intensity was observed in dendrites (apical or basal). See one example of the CA1 region of each genotype in Figure $5 A$. Sections from similar rostrocaudal 

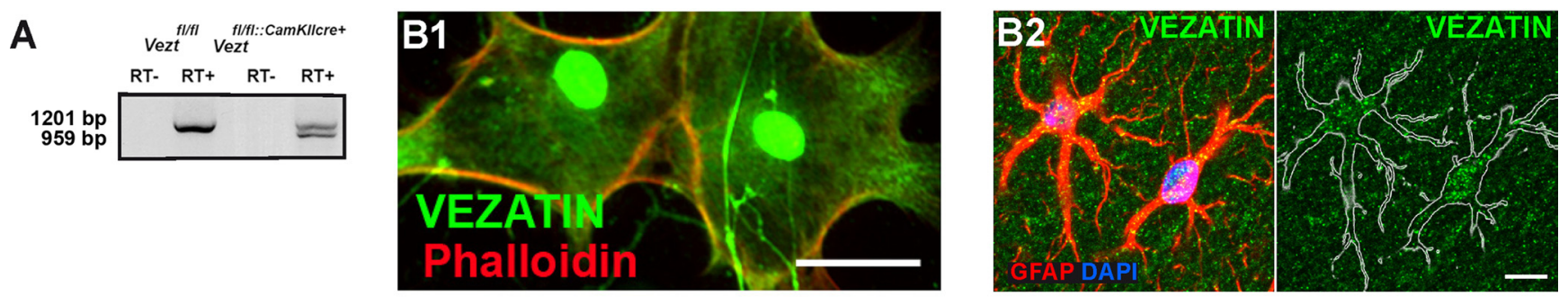

C


Figure 4. Vezatin expression is markedly extinguished in CamKI/cKO hippocampus in vivo. $\boldsymbol{A}$, The RT-PCR band deleted of exon 5 (959 bp) is detected in the cKO (Vezt/ffl::CamKI/cre + ) and not in the control mRNA (1201 bp band only), validating the proper cre-induced recombination. $B$, C, Vezatin is expressed by numerous cells in brain. $B 1$, Astrocytes, immunocytochemistry (phalloidin staining highlights astrocytic edges) of floxed/floxed astrocytes in long-term culture. B2, Confocal microscopy of Vezatin + GFAP + astrocytes in CA1. Scale bars: (in B1) $10 \mu \mathrm{m}$; (in B2, right) $20 \mu \mathrm{m}$. C, Interneurons, confocal microscopy of Vezatin + GAD65 + interneurons in CA1. CA1 region selected (arrow) for magnification (Right). Vezatin (green) and/or GAD65 (red), MAP2 (light blue), or DAPI (dark blue) are overlaid. Arrowheads point to two Vezatin + GAD65 + interneurons. Scale bar, $10 \mu \mathrm{m}$. D, Vezatin expression is decreased $\sim 60 \%$ using the CamKIlcre line. Representative cre $-/ c r e+$ paired images acquired in parallel under identical conditions (objective $40 \times$ and zoom). Vezatin staining is decreased in soma (SP) and dendritic areas in which synapses are located (SR) (see text for quantification). Arrowhead points to one example of Vezatin-expressing interneurons perfectly matching the confocal plane. MAP2 staining labels dendrites. Scale bars, $20 \mu \mathrm{m}$. SP, stratum pyramidale. Control, Vezt ${ }^{f / f l}$; CamKIl/cKO, Vezt ${ }^{f / f l}:$ :CamKIlcre+.

levels of CamKIIcre and control hippocampi were examined under light microscopy using a piezo-Z motor objective. A total of 37-40 secondary dendritic segments of homogeneous staining intensity were examined from 4 to 7 pyramidal neurons using Imaris built-in tools. Dendrites were semiautomatically tracked as a blue cylinder, and Golgi-Cox stains were manually indicated with a red spot under interactive 3D visualization. Red spots were categorized into two types. Spots lying on or touching the edge of the blue cylinder (i.e., without a clear neck) were classified as stubby spines. Spots away from the filament but within a margin of $3 \mu \mathrm{m}$ from the edge of the cylinder were classified as spines. This last category combined thin and mushroom spines. The extremely rare filopodia were excluded from the analysis. Spots out of the limit of $3 \mu \mathrm{m}$ from the cylinder edge were considered as not belonging to the tracked filament and excluded from the analysis. Examples of a tracked cylinder, selected/excluded spots
(B1, B3), and surface rendering (B2, B4) from each of the two genotypes are shown in Figure 5.

A total of 1302 and 1487 spots on a dendritic length of 1132.8 and $1233.7 \mu \mathrm{m}$ from control and CamKIIcKO, respectively, were categorized in either spines (combining thin and mushroom) or stubby spines that were subsequently counted in blind scoring conditions. The total density of spines + stubby spines was not significantly different in neurons from CamKIIcKO (12.05 spines/10 $\mu \mathrm{m})$ and control brains (11.93 spines/10 $\mu \mathrm{m})(p=0.3762$; unpaired $t$ test with Welch's correction). However, the number of stubby spines was significantly higher in CamKIIcKO than in control neurons (stubby spines $=25.4 \pm 1.6 \%$ and $14.3 \pm 1.8 \%$ in CamKIIcKO and control, respectively; unpaired Student's $t$ test; $p<0.00001$ ). The relatively higher proportion of stubby spines versus spines in mutant compared to control neurons suggests that Vezatin plays a role in spine formation and/or maturation. 

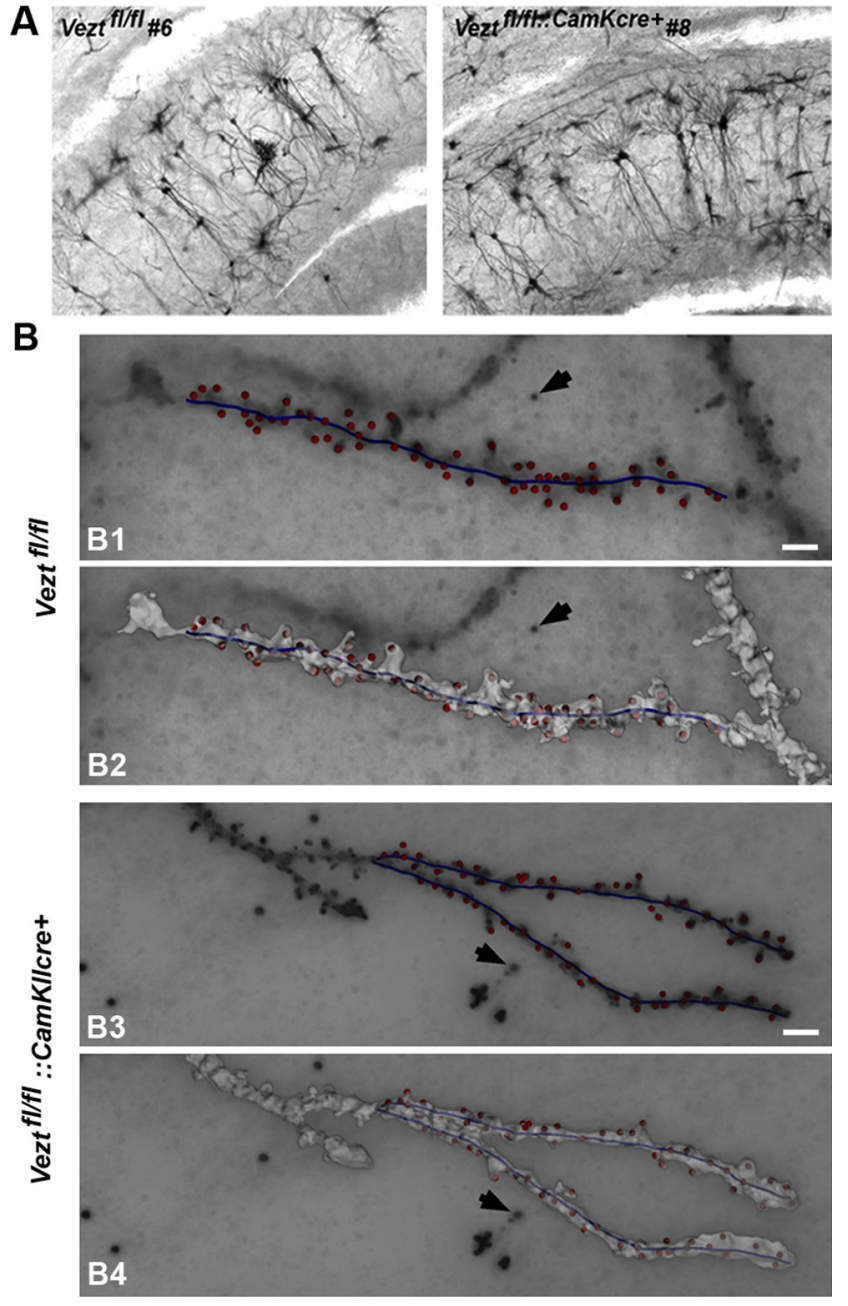

Figure 5. Increased number of stubby spines in CamKII/CKO (Vezt ${ }^{f l f l}::$ CamKIlcre +$)$ hippocampi in vivo. $\boldsymbol{A}$, CA1 dendrites are similarly stained after Golgi-Cox impregnation. $\boldsymbol{B}$, Digital images of representative Golgi-impregnated control and CamKI/CKO dendrites. B1, B3, Snapshot of 3D volumetric stack views using the FilamentTracer and Spot detection modules of the Imaris software package. Dendrites are semiautomatically tracked as blue cylinder by the filament tracer module. Golgi-Cox stains are indicated by red spots. $\boldsymbol{B} \mathbf{2}, \boldsymbol{B} \mathbf{4}$ Surface area rendering. Arrows (B1-B4) point to examples of excluded Golgi-Cox-stained spots. Scale bars (in B1 and B3), $3000 \mathrm{~nm}$

\section{Decreased anxiety- and memory-related behaviors in CamKIIcKO mice}

Because hippocampal spines are associated with important cognitive functions, we examined exploratory, anxiety-like, and memory performances in Vezt $t^{f l / f l}::$ CamKIIcre $+($ CamKIIcKO $)$ versus Vezt $t^{f l f l}$ (control) male mice.

Mice were tested for exploratory behavior in an open field. Habituation was normal in both genotypes. There was no significant difference in most of the parameters tested (total distance traveled in horizontal locomotion, distance traveled in the center or vertical rearing). However, CamKIIcKO mice showed a slight (16\%) but significant increased thigmotaxis (distance traveled at the periphery) as compared to control mice in the first of the two test sessions (Fig. 6A) (unpaired Student's $t$ test, $p<0.05$ ). This result suggests that there is a slight elevated exploratory and locomotor drive in CamKIIcKO mice.

We explored anxious behavior in the elevated plus maze. CamKIIcKO animals entered more often (unpaired Student's $t$ test, $p<0.01$ ) (Fig. 6B1) and spent more time in the open arms $(p<0.05)$ (Fig. 6B2), which resulted in a significant enhancement in the percentage of time spent in the open arms $(p<0.05)$ (Fig. 6B3). Additionally, CamKIIcKO mice displayed a significant increase in number of entries in the four arms $(p<0.05)$. These results suggest that CamKIIcKO mice are less anxious and/or less inhibited than control mice.

Nonspatial memory was tested in the passive avoidance apparatus. CamKIIcKO and control mice exhibited the same latency to cross to the dark side of the footshock-paired chamber during the training (Mann-Whitney $U$ test, $p=0.323$ ) and the test sessions, although a trend to forget the aversive association was noted in CamKIIcKO animals but not in controls $(p<0.075$ in the retention session) (Fig. 6C). This suggests that CamKIIcKO are not deficient in memory in this paradigm.

The MWM test was used to study spatial learning and relearning memory. Performances revealed no sensorimotor/ motivational abnormalities or learning/relearning deficits in CamKIIcKO (Fig. 6D). In the first and second probe trials, both groups of mice showed a preference and spent more time for/in the target quadrant (NE-first/SW-second) compared to the other three quadrants ( $p<0.01$ for the two groups) as well as for the target quadrant in the former acquisition phase (NE) after relearning ( $p<0.01$ for the two groups) (Fig. $6 D$, right, top/bottom). There was no difference in the latency to enter the target quadrant, the ghost platform, or the number of platform crossings. This indicates that there is no spatial learning or memory/flexibility impairment in CamKIIcKO mice in the MWM paradigm.

Finally, the role of Vezatin in learning/memory was explored in fear conditioning. Before the tone-shock pairs and during the acquisition phase, the level of freezing was similar in control and CamKIIcKO mice (10-20\%). This indicates that acquisition of fear conditioning of an unconditional stimulus (context/tone) with an aversive stimulus (electrical shock) is not impaired in CamKIIcKO mice. During contextual testing conducted $24 \mathrm{~h}$ after conditioning, the two groups froze to a similar extent (Fig. $6 E$ ), but when the conditioned stimulus (tone) was delivered in an altered context $48 \mathrm{~h}$ after conditioning (cued testing), the CamKIIcKO mice showed significantly less freezing than control mice $(p<0.05)$ (Fig. $6 F)$.

Together, these results indicate that our mutant mice showed decreased anxiety and/or disinhibition and a specific memory impairment in cued fear conditioning.

\section{Hippocampal synapses are maintained in $\mathrm{D} 6 \mathrm{cKO}$ dissociated neurons in vitro}

Since postnatal CamKIIcre expression does not allow us to examine further the role of Vezatin in spine morphogenesis during neuronal differentiation, we chose to study neurons from Vezt $t^{f l / f l}:: D 6 c r e+(D 6 c K O)$ embryos in which the Cre recombinase is expressed before birth, specific for hippocampus and cortex in adulthood and restricted to pyramidal and granule cells in the adult hippocampus (van den Boot et al., 2002). The recombination event was validated at the mRNA level (Fig. 7A). To first verify that Vezatin immunoreactivity was only suppressed in neurons and not in astrocytes, which perform many supporting functions in neuronal cultures, we used $\mathrm{N}$-cadherin together with Vezatin to visualize intercellular junctions in astrocytes in culture. Vezatin was normally codistributed with $\mathrm{N}$-cadherin at adherens junctions in $D 6 c K O$ cultured astrocytes at confluence as in control astrocytes (Fig. $7 B$ ). This indicates that glial cells express Vezatin similarly in cKO and control astrocytes. Additionally, we performed immunocytochemistry for a glial marker, GFAP, on 

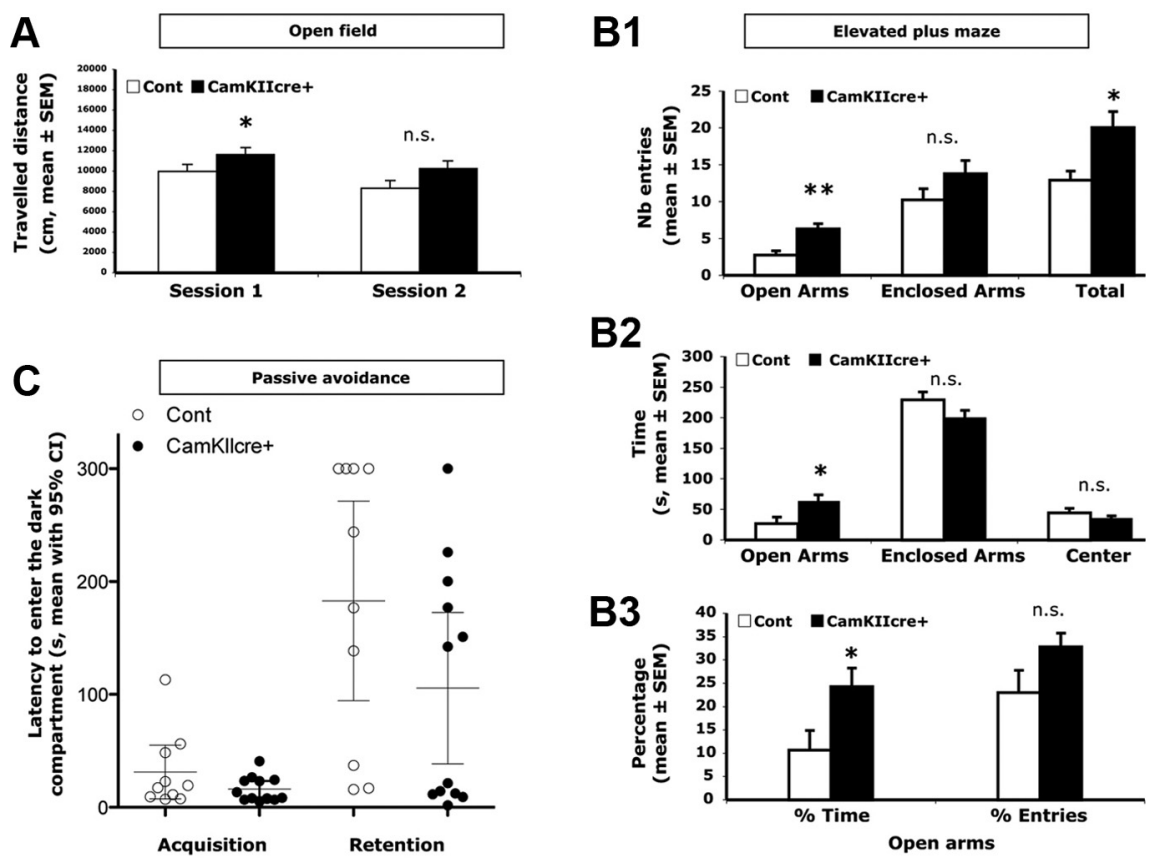

\section{B2}
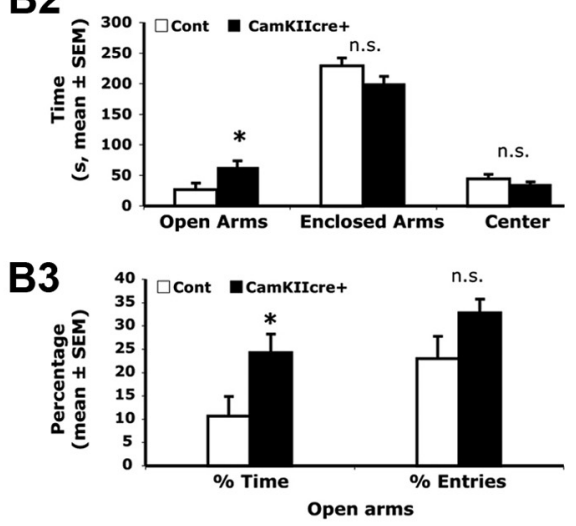

D Morris water maze


Probe test 2

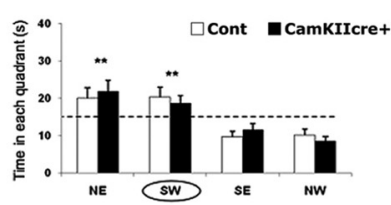

$\mathbf{E}$
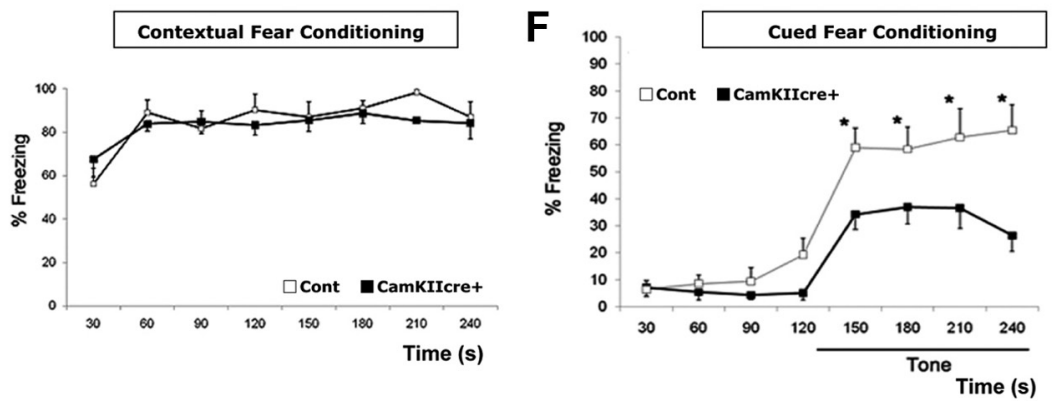

Figure 6. Decreased inhibition/anxiety-like behavior and impaired cued fear memory in CamKI/cK0 $\left(\right.$ Vezt $\left.{ }^{f l / f l}:: C a m K I I c r e+\right)$ mice. $A$, CamKIIcKO mice show slightly more exploratory behavior than control mice in horizontal exploration. It is significant in the first session $\left({ }^{*} p<0.05\right)$ and the trend is reproducible in the second session $(p=0.054)$ (Student's $t$ test). Bars indicate mean ( \pm SEM) distance traveled in centimeters in each of the two sessions. $\boldsymbol{B}$, CamKI/cKO mice are more anxious in the elevated plus maze. $\boldsymbol{B 1}$, Number of entries in open, enclosed, and all arms (mean \pm SEM). CamKI/cKO mice present a significant increase in the number of entries in all arms ( ${ }^{*} p<0.05$ ). B2, Time spent (in seconds) in open/enclosed arms and center of the maze (mean \pm SEM). CamKI/cKO mice present a significant increase in time spent in open arms $\left({ }^{* *} p<0.01\right)$. B3, Ratio of time spent and number of entries in open arms. CamKI/cKO mice are less anxious than control mice $\left({ }^{*} p<0.05\right)$ since they spent significantly more time in open arms. They also tend to enter more frequently into open arms ( $p=0.086$ ) (Student's $t$ test). C, No significantly altered memory in CamKllcK0 mice in passive avoidance test. Bars indicate latency (in seconds, median \pm quartiles) to enter the black compartment during acquisition/retention sessions, respectively. After $24 \mathrm{~h}$, CamKIIcK0 mice tend to cross sooner (i.e., to forget the noxious environment) ( $p=0.075$, Mann-Whitney $U$ test). $D$, No difference in path length in the three different protocols used in MWM (in centimeters): cued platform test, spatial learning, and spatial relearning (sum of two consecutive trials). Probe tests 1 and 2: both control and CamKIlcKO mice showed a significant preference for the correct quadrant during the two probe tests after learning and relearning $\left({ }^{* *} p<0.01\right)$ and still show a preference to the first brain adult sections showing comparable distribution of astrocytes in both genotypes ( $n=2$ pairs of cre-/cre + mice) (Fig. 7C). In hippocampus, no cellular layer disorganization was observed. Vezatin immunoreactivity was markedly diminished in soma and dendrites of $\mathrm{D} 6 \mathrm{cKO}$ versus control adult brain sections (Fig. $7 D, E$, CA1 field at higher power resolution). Vezatin staining in $\mathrm{cKO}$ was reduced by $53.3 \pm 2.75 \%$ compared to control [Vezt/MAP2 ratio $=0.0945$ au in cKO ( $n=146$ dendritic branches) and 0.2026 au in control ( $n=290$ branches); $p$ $<0.0001$, Mann-Whitney $U$ test; $n=2$ pairs of cre-/cre + mice). These results indicate that Vezatin expression is significantly reduced in adult hippocampal D6cKO neurons.

We characterized synaptic contacts in dissociated hippocampal neurons from D6cKO mice at 20-21 DIV by immunocytochemistry. Combinations of markers included Syb2, Synapsin1/2 (Syn) (vesicular proteins), and Bassoon (scaffolding protein) as presynaptic proteins. MAP2 was used to visualize the dendritic tree. Vezatin was included as a control for protein extinction. Presynaptic terminals labeled with Syb2 (Fig. $8 A, B$, top) or Bassoon (Fig. $8 B$, bottom) showed normal appearance on $\mathrm{D} 6 c \mathrm{KO}$ as on control dendrites. However, combined phalloidin and Syn staining showed that most synaptic contacts were flattened in the absence of Vezatin (Fig. 8C, compare D6cKO with control spine). To determine whether synaptic contacts were indeed present in the absence of Vezatin, we used Syn as a presynaptic marker together with PSD95 as a postsynaptic marker to label both sides of the synapse in the presence and absence of Vezatin (Fig. 8D). We blind scored the number of pre-postsynaptic appositions for the same dendritic length (at least 240 Syn + and PSD95 + spots) in control and D6cKO neurons. This number was not significantly different in D6cKO (D6 Cre+ ) compared to the number in control (D6 Cre -$)$ neurons $(p=$ 0.3727 ; unpaired $t$ test with Welch's correction) (Fig. $8 E$ ). This also indicates that

$\leftarrow$

position in the second probe test $\left.{ }^{* *} p<0.01\right)$. $\boldsymbol{E}$, Contextual fear conditioning responses displayed by CamKI/cKO and control mice are not significantly different $(p=0.9655)$. $\boldsymbol{F}$, During the first $2 \mathrm{~min}$, in the absence of the tone but in a different context, no freezing occurs in all mice showing that the different context is not inducing a fear response by itself. As soon as the tone is delivered during the next $2 \mathrm{~min}$, the percentage of time freezing ( \pm SEM) cued (tone) induced is significantly lower in CamKIlcKO mice compared to control mice ${ }^{*} p<0.05$ ). 
A
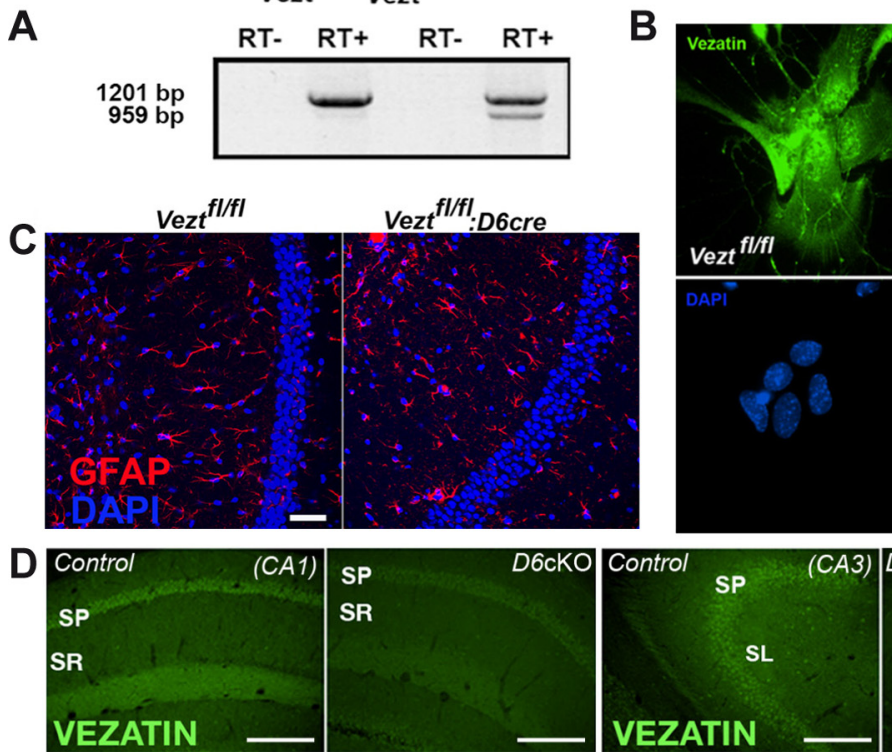

E

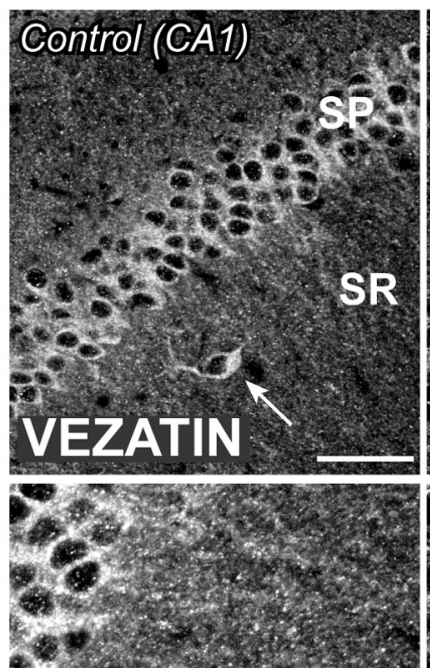

B

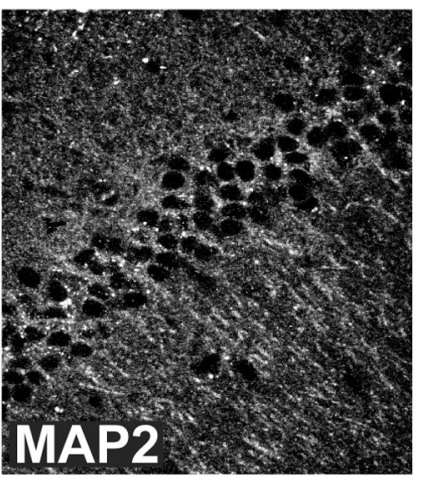

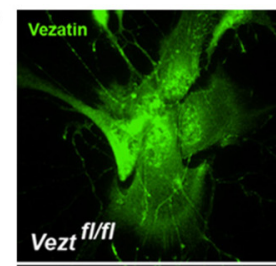
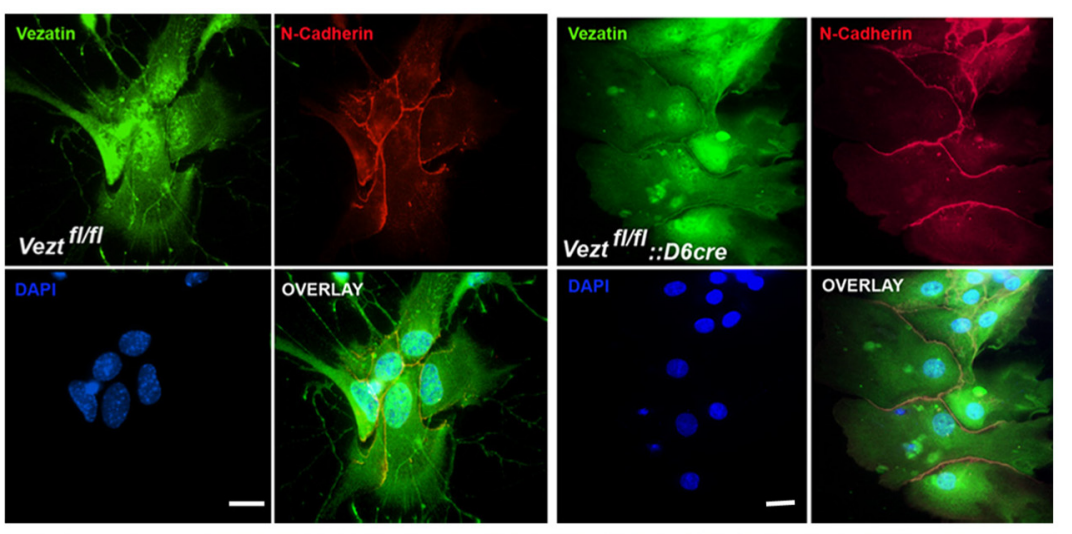

CKO

SP

SL
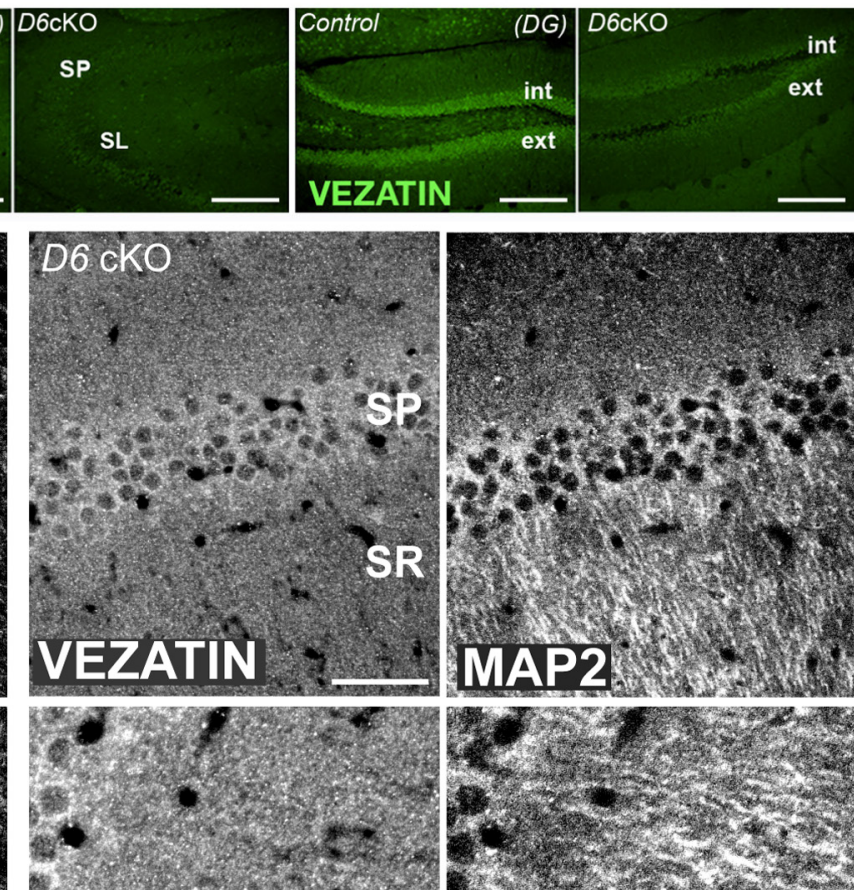

Figure 7. Vezatin expression is markedly extinguished in D6cKO hippocampus. A, The recombined RT-PCR band is present in D6cKO (Vezt ${ }^{f / f l}:: D 6 c r e+, 959$ bp) but not in control (Vezt ${ }^{f / f l}, 1201$ bp only) mRNA validating the proper cre recombination. $\boldsymbol{B}$, Vezatin is expressed in D6cKO (Vezt ${ }^{f / f l}:: D 6 c r e+$, right) and in control (Vezt $t^{f / f t}$, left) astrocytes in cultures at confluence colocalizing with $\mathrm{N}$-cadherin at adherens junctions (overlay). Scale bars, $5 \mu \mathrm{m}$. C, Numerous GFAP + astrocytes are similarly distributed in cKO (Vezt $t^{f / f l}::$ D6cre) and control (Vezt $\left.{ }^{f / f f}\right)$ sections. Scale bars: $10 \mu \mathrm{m}$. DAPI label nuclei $(\boldsymbol{B}, \boldsymbol{C}) . \boldsymbol{D}, \boldsymbol{E}$, Vezatin expression is diminished $\sim 50 \%$ in $D 6 c K 0$ neurons versus controls. Representative cre $-/ \mathrm{cre}+$ paired images acquired in parallel under identical conditions. $\boldsymbol{D}$, At low-power resolution (objective $20 \times$ ). Scale bar, $80 \mu \mathrm{m}$. $E$, At higher power resolution (objective $40 \times$ and zoom). The reduction is visible in cKO soma (SP) and dendrites where connections are located (SR) (see text for quantification). MAP2 staining labels dendrites. Arrow points to a Vezatin-expressing interneuron perfectly matching the confocal plane. Scale bars, $10 \mu \mathrm{m}$. SP, stratum pyramidale; SL, stratum lucidum; int, internal; ext, external. Control, Vezt ${ }^{f / f l}$; D6cK0, Vezt $t^{f / f f l:: D 6 c r e+}$.

Vezatin knock-out does not lead to a loss of PSD95 in the population of Syn+/Vezt - synapses in D6cKO neurons.

Together, our data indicate that postsynaptic sites remained tethered to presynaptic terminals in Vezatin-deficient hippocampal neurons. This suggests that Vezatin plays a role in the maturation rather than in the formation of synaptic contacts.

\section{Altered glutamatergic signaling at hippocampal synapses in} D6cKO mice in vivo

Most excitatory synapses in hippocampal neurons are established onto dendritic spines. We thus asked whether alterations in spine morphology upon reduction of Vezatin expression may induce a functional defect at excitatory synapses. To test this hypothesis, we performed an electrophysiological analysis using whole cell patch-clamp recordings in acute hippocampal slices of 3-week- old $D 6 c K O(D 6 c r e+)$ and control (D6cre-) mice. The frequency and amplitude of mEPSCs recorded from CA1 pyramidal cells were not significantly different between $\mathrm{D} 6 \mathrm{cKO}$ and control mice $[0.54 \pm 0.09$ vs $0.56 \pm 0.10 \mathrm{~Hz}(p=0.7)$ and $10.3 \pm 0.5$ vs $9.7 \pm$ $0.5 \mathrm{pA}(p=0.4) ; n=10$ and 13 cells, respectively], suggesting that both the number and the efficacy of functional synapses that contribute to mEPSCs were unaffected (Fig. 9A). This confirms that hippocampal spines in $\mathrm{D} 6 \mathrm{cKO}$ mice display functional transmission, despite their abnormal morphology.

Spine morphology has often been correlated with postsynaptic receptor content (Takumi et al., 1999). Small, potentially silent spines may contain only or mainly NMDARs whereas larger, more mature spines express both AMPARs and NMDARs. Thus, AMPAR/NMDAR ratio is often used as an index of synaptic maturity (Wu et al., 1996). We therefore compared the AMPAR/ 
A



B

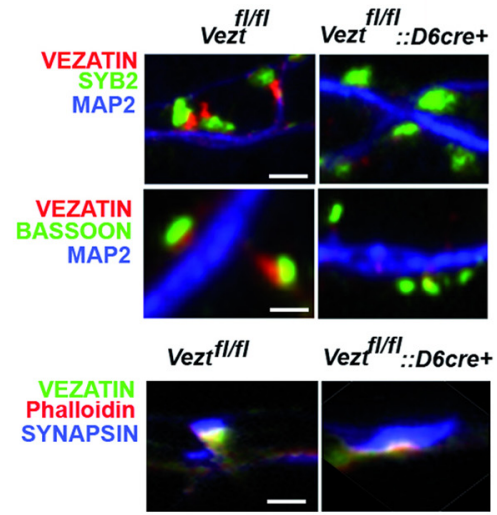

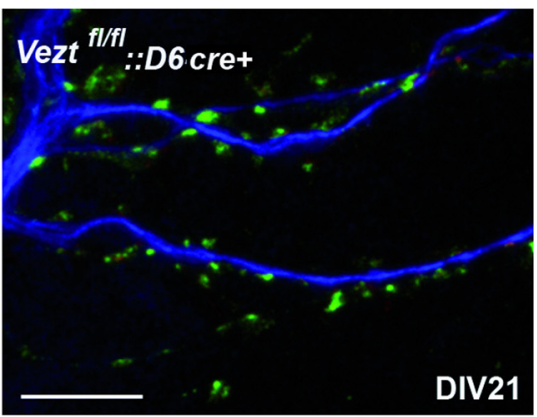

D

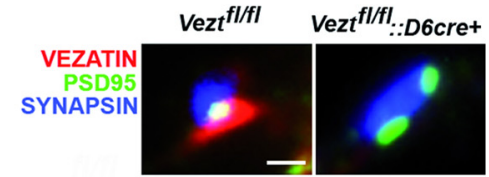

E

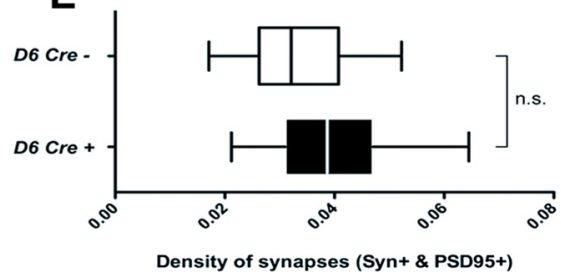

Figure 8. Reduced expression of Vezatin does not lead to loss of pre-postsynaptic appositions in D6cK0 neurons. A, Presynaptic Syb2 + signals are still present on dendrite from a representative D6cKO neuron. MAP2 is used to visualize the dendritic tree. Scale bars, $10 \mu \mathrm{m}$. B , Top, Syb2+ contacts at higher magnification. Bottom, Bassoon + signals are also present along dendrites in D6cK0 as in control neurons (at high magnification). C, Left, Phalloidin + Vezatin + spine from a control neuron has a mushroomlike shape typical of a mature spine and is facing Synapsin, a presynaptic marker, attesting to a synaptic contact. Right, By contrast, a phalloidin + Vezatin - spine from a D6cKO neuron is flattened. Interestingly, the phalloidin + Vezatin - spot is facing a Synapsin signal, suggesting that a synapse is present even in stubby spines (see below). D, Top, PSD95 + spine is en face Synapsin in D6cKO (right), as in control neurons (left). $\boldsymbol{E}$, Quantification of the number of synaptic contacts exemplified in $\boldsymbol{D}$. Statistical analysis shows that the density of Synapsin + PSD95 + appositions on dendritic segments is not significantly different. Scale bars $(B-D), 2 \mu \mathrm{m}$.

NMDAR ratio at Schaffer collateral synapses between $\mathrm{D} 6 \mathrm{KO}$ and control mice. AMPAR- and NMDAR-mediated EPSCs were recorded while holding cells at +40 and $-60 \mathrm{mV}$, respectively. As illustrated in Figure $9 B, C$, the AMPAR/NMDAR current ratio was significantly reduced in the $D 6 c K O(D 6 c r e+)$ compared to the control $(D 6 \mathrm{cre}-)$ mice $(2.41 \pm 0.35$ vs $3.77 \pm 0.73 ;-36 \%$; $p<0.05$, Mann-Whitney rank sum test; $n=16$ and 10 cells, respectively). These results suggest that Vezatin, in addition to its role in spine morphology also influences synaptic functional maturation in hippocampal neurons.

Evoked EPSCs showed reduced AMPAR/NMDAR current ratio. To examine whether this was due to a reduced number of postsynaptic AMPARs, we quantified AMPAR + clusters in the SR of D $6 c K O$ and controls using polyclonal antibodies to the extracellular domain of GluA2, the most prevalent subunit in AMPARs. Consistent with electrophysiological results (mEPSCs), we found no significant difference in the number of GluA2-AMPAR in $D 6 c K O$ compared to control hippocampi ( $p=0.0660$, MannWhitney $U$ test).

\section{Discussion}

We show here that Vezatin is expressed in spines, the postsynaptic compartment of excitatory spiny neurons. At the cellular level, significant reduction of Vezatin is associated with spine flattening. We further demonstrate that the reduced level of neuronal Vezatin affects behavior and functional synaptic maturation.
Vezatin associates with actin at epithelial cell-cell junctions via proteins containing a FERM (F, 4.1 protein; E, ezrin; R, radixin; $M$, moesin) domain (Myosin $7 \mathrm{a}$, Ezrin/Radixin), thus contributing to the generation of cellular mechanical tension between the actin cytoskeleton and the plasma membrane (Kussel-Andermann et al., 2000; Bahloul et al., 2009). In the inner ear, Vezatin also belongs to a molecular complex that critically mediates the early cohesion of hair bundles, the mechanoreceptor structure essential to the auditory function (Michalski et al., 2007). Here, we propose that Vezatin could play a role in shape/tension regulation of the spine. First, Vezatin is at the margin of the PSD (Fig. $2 B, C$ ) and the base of the spine head (Fig. 2 E). Interestingly, Myo IIB, an actomyosin contractile motor important for generating tangential forces at the spine head membrane, and Cofilin, a severing actin-binding protein, also concentrate at the edges of the PSD (Ryu et al., 2006; Racz and Weinberg, 2006). It was shown that Myo IIB elimination generates protrusion-like spine increase (Ryu et al., 2006; Rex et al., 2010) and that Cofilin inhibition/elimination induces spine shrinkage (Gu et al., 2010; Zhou et al., 2004; Rust et al., 2010). Furthermore, the periphery and base of the head are associated with F-actin dynamic/constriction important for spine maturation/maintenance, respectively (Halpain, 2000; Roelandse et al., 2003; Honkura et al., 2008). Second, we observed that Vezatin expression extends within the neck (Fig. $2 B, C$ ); this geometry is important for synaptic plasticity and actin fiber confinement (Noguchi et al., 2005; Honkura et al., 2008). Finally, Vezatin is expressed at the $T$ zone of growth cones enriched in buckling F-actin and Myo IIB (Fig. 1E). The T zone participates in linking growth cone tensile forces to actin dynamics (Medeiros et al., 2006; Lowery and Van Vactor, 2009). Despite the limited resolution of light microscopy, our present observations are thus suggestive of a role of Vezatin in the dynamics of the spine shape (see below).

As expected for a protein important in spine morphogenesis, we show here that reducing Vezatin expression results in an increase in stubby spines. Stubby spines do not have a clear neck and are closely connected to the dendritic shaft (Harris et al., 1992; Bourne and Harris, 2008). They account for more than half the total number of protrusions in young cultured neurons (Parnass et al., 2000) and are observed following various experimental protocols (McKinney et al., 1999, Hasbani et al., 2001a, 2001b; Jourdain et al., 2002). Interestingly, actin depolymerization in hippocampal neurons induces a loss of AMPAR clusters, and the remaining clusters appeared on small deflated structures (Allison et al., 1998). We previously showed that Vezatin elimination induces a destabilization of the adhesion complex (Hyenne et al., 2005) and participates to the maturation rather than the formation of cellular junctions in epithelial cells (Bahloul et al., 2009). Based on previous works in epithelial cells and our present data, we suggest that spine flattening in Vezatin-deficient hippocampal 


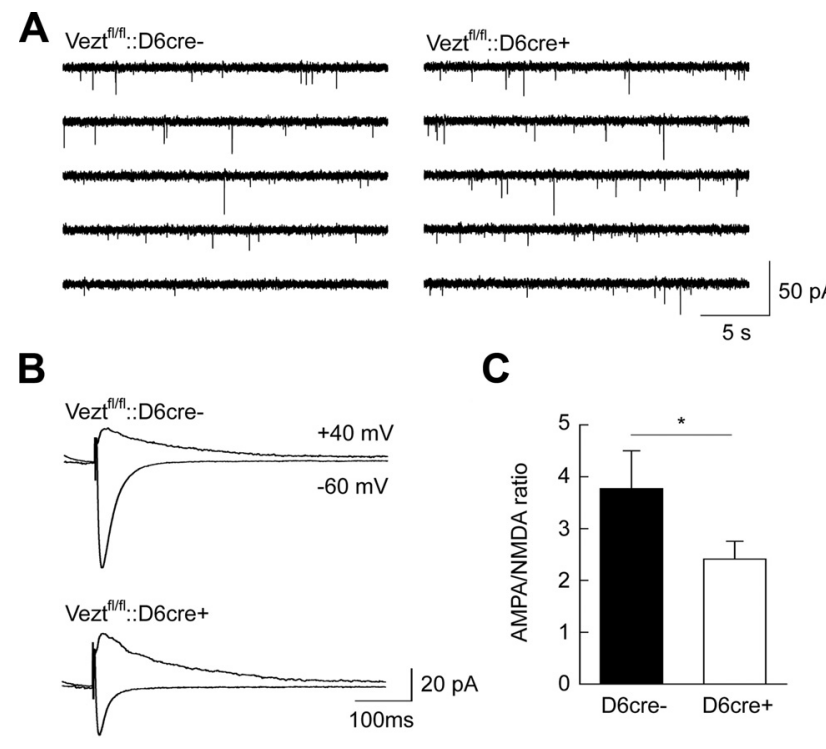

Figure 9. Functional alteration of synaptic function induced by reduced expression of Vezatin expression at hippocampal excitatory synapses. $A, \mathrm{mEPSC}$ recorded from CA1 pyramidal neurons in 3-week-old Vezt ${ }^{f / f l}::$ D6cre - (control, top) and Vezt ${ }^{f / f l}::$ D6cre + (D6cKO, bottom) littermates. Representative $120 \mathrm{~s}$ continuous recordings are shown from each condition. $\boldsymbol{B}$, Evoked EPSCs recorded from CA1 pyramidal cells on stimulation of the Schaffer collaterals in 3-week-old Vezt ${ }^{f / f l}:: D 6 c r e-\left(\right.$ control, top) and Vezt ${ }^{f / f f}::$ :D6cre $+(D 6 c K 0$, bottom) mice. Each trace represents the average of 50 consecutive EPSCs recorded at $0.2 \mathrm{~Hz}$ while holding cells at either -60 or $+40 \mathrm{mV}$. C, AMPA/NMDA ratios averaged from 10 and 16 independent recordings from control $(D 6 \mathrm{cre}-)$ and $D 6 c \mathrm{~K} 0(D 6 \mathrm{cre}+)$ mice, respectively. The AMPA/NMDA ratio was significantly lower $(p<0.05)$ in D6cre + vs D6cre - mice.

neurons is due to a maturation defect of the postsynaptic compartment.

Flattened spines after intense stimulation or actin depolymerization remain tethered to presynaptic boutons (Hasbani et al., 2001a). This indicates that retracted spines may not lose a synaptic connection. We show here that Vezatin mutant neurons still receive functional excitatory inputs with unaffected frequency and amplitude. This indicates that a reduction of Vezatin expression in spines does not compromise synaptic function despite abnormal morphology. However, we cannot exclude a differential/compensatory alteration of synaptic function at flattened versus normal spines in cKO mice. Additionally, we cannot exclude that residual expression is sufficient to support the remaining synaptic function.

Evoked EPSCs showed reduced AMPAR/NMDAR ratio, a classical index of synaptic maturity in a variety of neurons (Wu et al., 1996; Isaac et al., 1997). Since the mean quantal size of AMPAR mEPSCs is unchanged in Vezatin mutant slices, this result is unlikely to reflect a reduced number or function of postsynaptic AMPAR. Accordingly, we found a similar number of GluA2-AMPARs in mutant and control brains. Instead we thus suggest that the reduced AMPAR/NMDAR ratio may reflect either a greater proportion of silent synapses or enhanced function/number of postsynaptic NMDAR. Silent synapses contain NMDAR but are devoid of functional AMPAR and thus do not mediate synaptic transmission at rest (Poncer, 2003). Activity-dependent insertion of AMPAR at silent synapses is a proposed substrate for long-term synaptic plasticity and maturation (Bourne and Harris, 2008). In CA1 hippocampal neurons, functional conversion of silent synapses occurs during early postnatal development (Durand et al., 1996; Petralia et al., 1999) and requires basal neuronal activity and NMDAR activation (Zhu et al., 2000). Since activitydependent delivery of AMPAR is dependent on dynamic actin cytoskeleton (Kim and Lisman, 1999; Krucker et al., 2000), we suggest that reduced expression of Vezatin in dendritic spines might compromise functional conversion of silent synapses. Alternatively, we cannot exclude that postsynaptic NMDAR function and/or number was enhanced on the reduced expression of Vezatin, leading to a reduced AMPAR/NMDAR ratio. In fact, a specific increase in NMDAR-mediated transmission was recently observed on genetic ablation of the adaptor protein IRSp53 involved in actin filament assembly (Kim et al., 2009). Further experiments will be needed to fully characterize these functional deficits as well as their consequences on synaptic maturation and plasticity at hippocampal excitatory synapses.

Yet, consistent with a function of Vezatin in spine maturation, we further observed an impairment in associative aversive memory and a decreased response in anxiety-related task that may be related to a disinhibition behavior. The hippocampus is believed to integrate a spatial environment (Bird and Burgess, 2008) or a contextual complex representation (Maren, 2001). Interestingly, although certainly oversimplified, septal (dorsal) and temporal (ventral) poles of the hippocampus would encode spatial- and fear-associated information, respectively (Bannerman et al., 2002; Kjelstrup et al., 2002; Maren and Holt, 2004; Adhikari et al., 2011). In addition, a prominent role of temporal hippocampus and amygdalo-hippocampal connections has been described in fear-conditioning memory to tone (Maren and Holt, 2004). CamKIIcKO mutant mice in which Vezatin was inactivated after the main brain developmental events did not show any deficit in spatial memory or in contextual fear conditioning. The lack of major hippocampal-related behavioral defect could be due to compensatory mechanisms, to a different susceptibility of the neuronal circuits to various behavioral tests, or to incomplete ablation of Vezatin expression. Nevertheless, mutant mice are less inhibited/ anxious and have a reduced fear response to tone versus controls. Since no gross auditory defect was detected (Preyer reflex), this indicates that Vezatin-deficient mice have a memory defect in associating an elemental representation (a tone) to the aversive stimulus together with a decrease in anxiety-related response. Accordingly, Vezatin would be more critical to neural substrates in the temporal hippocampus than to circuits that require the septal hippocampus. Although Vezatin is poorly expressed in amygdala nuclei, Vezatin decreased expression in amygdala areas may also contribute to the fear-induced memory deficit.

Transsynaptic dialog between adhesion molecules is important in synaptogenesis and synaptic plasticity (Futai et al., 2007; Saglietti et al., 2007; Kim et al., 2008; Sudhof, 2008; Choi et al., 2011). Vezatin is an integral protein with two adjacent transmembrane domains and cytoplasmic N-/C-terminal regions (Bahloul et al., 2009), implying that it cannot directly mediate transsynaptic interactions. Moreover, Vezatin partitioned postsynaptically. Rather than a role in neuronal transmission through a transsynaptic interplay, our data suggest that Vezatin contributes to linking cytoskeleton dynamics and plasma membrane components, adjusting cellular tension during growth of the postsynaptic compartment. Still, Vezatin indirectly linked to $\mathrm{N}$-cadherin may form a transcomplex stimulating spine maturation/function (Saglietti et al., 2007).

Finally, Vezatin is one effector of the GTPase Arf6 (Sousa et al., 2005; Hyenne et al., 2007b; Sanda et al., 2010). Arf6 knockdown results in decreased spine density while Arf6-GTP overexpression promotes spine formation/maturation (Choi et al., 
2006). Sh-RNA-induced Vezatin knock-down of neurons at 5 DIV reduces dendritic length/arborization complexity, and an increase in dendritic length is observed following overexpression (Sanda et al., 2010). While our results fit well with findings that Arf6 negatively regulates spine maturation (Choi et al., 2006) and that Vezatin positively regulates dendritic arborization complexity (Sanda et al., 2010), no effect on dendritic length at 21 DIV was observed. Based on the data obtained using the refined genetic invalidation approaches shown here, we thus favor the hypothesis that Vezatin is a positive regulator of actin dynamics in spines and neurite branching but not a major regulator of dendritic growth. Alternatively, Vezatin may act as a negative regulator of stubby spine formation.

In conclusion, the present findings together with the involvement in adhesion in association with a myosin suggest that Vezatin may be a key element in remodeling dendritic spines by coupling tension between the membrane and the cytoskeleton.

\section{Notes}

Supplemental Movies 1 and 2 for this article are available at http://lydia. danglot.free.fr/Movie.html. This material has not been peer reviewed.

\section{References}

Adhikari A, Topiwala MA, Gordon, JA (2011) Single units in the medial prefrontal cortex with anxiety-related firing patterns are preferentially influenced by ventral hippocampal activity. Neuron 71:898 -910.

Allison DW, Gelfand VI, Spector I, Craig AM (1998) Role of actin in anchoring postsynaptic receptors in cultured hippocampal neurons: differential attachment of NMDA versus AMPA receptors. J Neurosci 18:2423-2436.

Ankri N, Legendre P, Faber DS, Korn H (1994) Automatic detection of spontaneous synaptic responses in central neurons. J Neurosci Methods 52:87-100.

Arikkath J, Reichardt LF (2008) Cadherins and catenins at synapses: role in synaptogenesis and synaptic plasticity. Trends Neurosci 31:487-494.

Bahloul A, Simmler MC, Michel V, Leibovici M, Perfettini I, Roux I, Weil D, Nouaille S, Zuo J, Zadro C, Licastro D, Gasparini P, Avan P, Hardelin JP, Petit C (2009) Vezatin, an integral membrane protein of adherens junctions, is required for the sound resilience of cochlear hair cells. EMBO Mol Med 1:125-138.

Bannerman DM, Deacon RM, Offen S, Friswell J, Grubb M, Rawlins JN (2002) Double dissociation of function within the hippocampus: spatial memory and hyponeophagia. Behav Neurosci 116:884-901.

Bemelmans AP, Bonnel S, Houhou L, Dufour N, Nandrot E, Helmlinger D, Sarkis C, Abitbol M, Mallet J (2005) Retinal cell type expression specificity of HIV-1-derived gene transfer vectors upon subretinal injection in the adult rat: influence of pseudotyping and promoter. J Gene Med 7:1367-1374.

Benson DL, Huntley GW (2011) Synapse adhesion: a dynamic equilibrium conferring stability and flexibility. Curr Opin Neurobiol 22:1-8.

Bird CM, Burgess N (2008) The hippocampus and memory: insights from spatial processing. Nat Rev Neurosci 9:182-194.

Bourgeron T (2009) A synaptic trek to autism. Curr Opin Neurobiol 19:231-234.

Bourne JN, Harris KM (2008) Balancing structure and function at hippocampal dendritic spines. Annu Rev Neurosci 31:47-67.

Burke SN, Barnes CA (2006) Neural plasticity in the ageing brain. Nat Rev Neurosci 7:30-40.

Casanova E, Fehsenfeld S, Mantamadiotis T, Lemberger T, Greiner E, Stewart AF, Schütz G (2001) A CamKIIalpha iCre BAC allows brain-specific gene inactivation. Genesis 31:37-42.

Choi S, Ko J, Lee H, Kim K, Chung H, Kim HS, Kim E (2006) ARF6 and EFA6A regulate the development and maintenance of dendritic spines. J Neurosci 26:4811-4919.

Choi YB, Li HL, Kassabov SR, Jin I, Puthanveettil SV, Karl KA, Lu Y, Kim JH, Bailey CH, Kandel ER (2011) Neurexin-neuroligin transsynaptic interaction mediates learning-related synaptic remodeling and long-term facilitation in aplysia. Neuron 70:468-481.

Danglot L, Triller A, Bessis A (2003) Association of gephyrin with synaptic and extrasynaptic GABAA receptors varies during development in cultured hippocampal neurons. Mol Cell Neurosci 2:264-278.

Durand GM, Kovalchuk Y, Konnerth A (1996) Long-term potentiation and functional synapse induction in developing hippocampus. Nature 381:71-75.

Fiala CJ, Spacek J, Harris KM (2008) Dendrite structure. In: Dendrites (Stuart G, Spruston N, Häusser M eds), pp 1-41. Oxford: Oxford UP.

Futai K, Kim MJ, Hashikawa T, Scheiffele P, Sheng M, Hayashi Y (2007) Retrograde modulation of presynaptic release probability through signaling mediated by PSD-95-neuroligin. Nat Neurosci 10:186-195.

Gibb R, Kolb B (1998) A method for vibratome sectioning of Golgi-Cox stained whole rat brain. J Neurosci Methods 79:1-4.

Glaser EM, Van der Loos H (1981) Analysis of thick brain sections by obverse-reverse computer microscopy: application of a new, high clarity Golgi-Nissl stain. J Neurosci Methods 4:117-125.

Gu J, Lee CW, Fan Y, Komlos D, Tang X, Sun C, Yu K, Hartzell HC, Chen G, Bamburg JR, Zheng JQ (2010) ADF/cofilin-mediated actin dynamics regulate AMPA receptor trafficking during synaptic plasticity. Nat Neurosci 13:1208-1215.

Halpain S (2000) Actin and the agile spine: how and why do dendritic spines dance? Trends Neurosci 23:141-146.

Harris KM, Stevens JK (1989) Dendritic spines of CA1 cells in the rat hippocampus: serial electron microscopy with reference to their biophysical characteristics. J Neurosci 9:2982-2997.

Harris KM, Jensen FE, Tsao B (1992) Three-dimensional structure of dendritic spines and synapses in rat hippocampus (CA1) at postnatal $\mathrm{d} 15$ and adult ages: implications for the maturation of synaptic physiology and long-term potentiation. J Neurosci 12:2685-2705.

Hasbani MJ, Schlief ML, Fisher DA, Goldberg MP (2001a) Dendritic spines lost during glutamate receptor activation reemerge at original sites of synaptic contact. J Neurosci 21:2393-2403.

Hasbani MJ, Viquez NM, Goldberg MP (2001b) NMDA receptors mediate hypoxic spine loss in cultured neurons. Neuroreport 12:2731-2735.

Hering H, Sheng M (2001) Dendritic spines: structure, dynamics and regulation. Nat Rev Neurosci 2:880-888.

Honkura N, Matsuzaki M, Noguchi J, Ellis-Davies GC, Kasai H (2008) The subspine organization of actin fibers regulates the structure and plasticity of dendritic spines. Neuron 57:719-729.

Hyenne V, Louvet-Vallée S, El-Amraoui A, Petit C, Maro B, Simmler MC (2005) Vezatin, a protein associated to adherens junctions, is required for mouse blastocyst morphogenesis. Dev Biol 287:180-191.

Hyenne V, Harf JC, Latz M, Maro B, Wolfrum U, Simmler MC (2007a) Vezatin, a ubiquitous protein of adherens cell-cell junctions, is exclusively expressed in germ cells in mouse testis. Reproduction 133:563-574.

Hyenne V, Souilhol C, Cohen-Tannoudji M, Cereghini S, Petit C, Langa F, Maro B, Simmler MC (2007b) Conditional knock-out reveals that zygotic vezatin-null mouse embryos die at implantation. Mech Dev 124:449-462.

Isaac JT, Crair MC, Nicoll RA, Malenka RC (1997) Silent synapses during development of thalamocortical inputs. Neuron 18:269-280.

Jourdain P, Nikonenko I, Alberi S, Muller D (2002) Remodeling of hippocampal synaptic networks by a brief anoxia-hypoglycemia. J Neurosci 22:3108-3116.

Kawabe H, Neeb A, Dimova K, Young SM Jr, Takeda M, Katsurabayashi S, Mitkovski M, Malakhova OA, Zhang DE, Umikawa M, Kariya K, Goebbels S, Nave KA, Rosenmund C, Jahn O, Rhee J, Brose N (2010) Regulation of Rap2A by the ubiquitin ligase Nedd4-1 controls neurite development. Neuron 65:358-372.

Kellendonk C, Tronche F, Monaghan AP, Angrand PO, Stewart F, Schütz G (1996) Regulation of Cre recombinase activity by the synthetic steroid RU 486. Nucleic Acids Res 24:1404-1411.

Kerchner G, Nicoll R (2008) Silent synapses and the emergence of a postsynaptic mechanism for LTP. Nat Rev Neurosci 9:813-825.

Kerr JM, Blanpied TA (2012) Subsynaptic AMPA receptor distribution is acutely regulated by actin-driven reorganization of the postsynaptic density. J Neurosci 32:658-673.

Kim CH, Lisman JE (1999) A role of actin filament in synaptic transmission and long-term potentiation. J Neurosci 19:4314-4324.

Kim J, Jung SY, Lee YK, Park S, Choi JS, Lee CJ, Kim HS, Choi YB, Scheiffele P, Bailey CH, Kandel ER, Kim JH (2008) Neuroligin-1 is required for normal expression of LTP and associative fear memory in the amygdala of adult animals. Proc Natl Acad Sci U S A 105:9087-9092. 
Kim MH, Choi J, Yang J, Chung W, Kim JH, Paik SK, Kim K, Han S, Won H, Bae YS, Cho SH, Seo J, Bae YC, Choi SY, Kim E (2009) Enhanced NMDA receptor-mediated synaptic transmission, enhanced long-term potentiation, and impaired learning and memory in mice lacking IRSp53. J Neurosci 29:1586-1595.

Kjelstrup KG, Tuvnes FA, Steffenach HA, Murison R, Moser EI, Moser MB (2002) Reduced fear expression after lesions of the ventral hippocampus. Proc Natl Acad Sci U S A 99:10825-10830.

Krucker T, Siggins GR, Halpain S (2000) Dynamic actin filaments are required for stable long-term potentiation (LTP) in area CA1 of the hippocampus. Proc Natl Acad Sci U S A 97:6856-6861.

Küssel-Andermann P, El-Amraoui A, Safieddine S, Nouaille S, Perfettini I, Lecuit M, Cossart P, Wolfrum U, Petit C (2000) Vezatin, a novel transmembrane protein, bridges myosin VIIA to the cadherin-catenins complex. EMBO J 19:6020-6029.

Law JW, Lee AY, Sun M, Nikonenko AG, Chung SK, Dityatev A, Schachner M, Morellini F (2003) Decreased anxiety, altered place learning, and increased CA1 basal excitatory synaptic transmission in mice with conditional ablation of the neural cell adhesion molecule L1. J Neurosci 23:10419-10432.

Lin B, Kramár EA, Bi X, Brucher FA, Gall CM, Lynch G (2005) Theta stimulation polymerizes actin in dendritic spines of hippocampus. J Neurosci 25:2062-2069.

Lowery L, Van Vactor D (2009) The trip of the tip: understanding the growth cone machinery. Nat Rev Mol Cell Biol 10:332-342.

Maren S (2001) Neurobiology of Pavlovian fear conditioning. Annu Rev Neurosci 24:897-931.

Maren S, Holt WG (2004) Hippocampus and Pavlovian fear conditioning in rats: muscimol infusions into the ventral, but not dorsal, hippocampus impair the acquisition of conditional freezing to an auditory conditional stimulus. Behav Neurosci 118:97-110.

McKinney RA, Capogna M, Dürr R, Gähwiler BH, Thompson SM (1999) Miniature synaptic events maintain dendritic spines via AMPA receptor activation. Nat Neurosci 2:44-49.

Medeiros NA, Burnette DT, Forscher P (2006) Myosin II functions in actinbundle turnover in neuronal growth cones. Nat Cell Biol 8:215-226.

Michalski N, Michel V, Bahloul A, Lefèvre G, Barral J, Yagi H, Chardenoux S, Weil D, Martin P, Hardelin JP, Sato M, Petit C (2007) Molecular characterization of the ankle-link complex in cochlear hair cells and its role in the hair bundle functioning. J Neurosci 27:6478-6488.

Noguchi J, Matsuzaki M, Ellis-Davies GC, Kasai H (2005) Spine-neck geometry determines NMDA receptor-dependent $\mathrm{Ca} 2+$ signaling in dendrites. Neuron 46:609-622.

Obiang P, Maubert E, Bardou I, Nicole O, Launay S, Bezin L, Vivien D, Agin V (2011) Enriched housing reverses age-associated impairment of cognitive functions and tPA-dependent maturation of BDNF. Neurobiol Learn Mem 96:121-129.

Parnass Z, Tashiro A, Yuste R (2000) Analysis of spine morphological plasticity in developing hippocampal pyramidal neurons. Hippocampus 10:561-568.

Penzes P, Cahill ME, Jones KA, VanLeeuwen JE, Woolfrey KM (2011) Dendritic spine pathology in neuropsychiatric disorders. Nat Neurosci 14:285-293.

Petralia RS, Esteban JA, Wang YX, Partridge JG, Zhao HM, Wenthold RJ, Malinow R (1999) Selective acquisition of AMPA receptors over postnatal development suggests a molecular basis for silent synapses. Nat Neurosci 2:31-36.

Poncer JC (2003) Hippocampal long term potentiation: silent synapses and beyond. J Physiol Paris 97:415-422.

Racz B, Weinberg RJ (2006) Spatial organization of cofilin in dendritic spines. Neuroscience 138:447-456.

Rex CS, Gavin CF, Rubio MD, Kramar EA, Chen LY, Jia Y, Huganir RL, Muzyczka N, Gall CM, Miller CA, Lynch G, Rumbaugh G (2010) Myosin IIb regulates actin dynamics during synaptic plasticity and memory formation. Neuron 67:603-617.

Roelandse M, Welman A, Wagner U, Hagmann J, Matus A (2003) Focal motility determines the geometry of dendritic spines. Neuroscience 121:39-49.

Rust MB, Gurniak CB, Renner M, Vara H, Morando L, Görlich A, SassoèPognetto M, Banchaabouchi MA, Giustetto M, Triller A, Choquet D, Witke W (2010) Learning, AMPA receptor mobility and synaptic plas- ticity depend on n-cofilin-mediated actin dynamics. EMBO J 29:18891902.

Ryu J, Liu L, Wong TP, Wu DC, Burette A, Weinberg R, Wang YT, Sheng M (2006) A critical role for myosin IIb in dendritic spine morphology and synaptic function. Neuron 49:175-182.

Saglietti L, Dequidt C, Kamieniarz K, Rousset MC, Valnegri P, Thoumine O, Beretta F, Fagni L, Choquet D, Sala C, Sheng M, Passafaro M (2007) Extracellular interactions between GluR2 and N-cadherin in spine regulation. Neuron 54:461-477.

Sanda M, Ohara N, Kamata A, Hara Y, Tamaki H, Sukegawa J, Yanagisawa T, Fukunaga K, Kondo H, Sakagami H (2010) Vezatin, a potential target for ADP-ribosylation factor 6, regulates the dendritic formation of hippocampal neurons. Neurosci Res 67:126-136.

Sandi C, Bisaz R (2007) A model for the involvement of neural cell adhesion molecules in stress-related mood disorders. Neuroendocrinology 85 : $158-176$.

Scheuber A, Rudge R, Danglot L, Raposo G, Binz T, Poncer JC, Galli T (2006) Loss of AP-3 function affects spontaneous and evoked release at hippocampal mossy fiber synapses. Proc Natl Acad Sci U S A 103:16562-16567.

Shapiro L, Love J, Colman DR (2007) Adhesion molecules in the nervous system: structural insights into function and diversity. Annu Rev Neurosci 30:451-474.

Sheng M, Kim MJ (2002) Postsynaptic signaling and plasticity mechanisms. Science 298:776-780.

Sheng M, Hoogenraad CC (2007) The postsynaptic architecture of excitatory synapses: a more quantitative view. Annu Rev Biochem 76:823-847.

Sholl DA (1953) Dendritic organization in the neurons of the visual and motor cortices of the cat. J Anat 87:387-406.

Sirven A, Pflumio F, Zennou V, Titeux M, Vainchenker W, Coulombel L, DubartKupperschmitt A, Charneau P (2000) The human immunodeficiency virus type-1 central DNA flap is a crucial determinant for lentiviral vector nuclear import and gene transduction of human hematopoietic stem cells. Blood 96:4103-4110.

Sousa S, Cabanes D, Archambaud C, Colland F, Lemichez E, Popoff M, Boisson-Dupuis S, Gouin E, Lecuit M, Legrain P, Cossart P (2005) ARHGAP10 is necessary for a-catenin recruitment at adherens junctions and for Listeria invasion. Nat Cell Biol 7:954-960.

Steel K, Hardisty R (1996) Assessing hearing, vision and balance in mice. In: What's wrong with my mouse? New interplays between mouse genes and behavior (Short Course Syllabus), pp 26-38. Washington, DC: Society for Neuroscience.

Südhof TC (2008) Neuroligins and neurexins link synaptic function to cognitive disease. Nature 455:903-911.

Takeichi M, Abe K (2005) Synaptic contact dynamics controlled by cadherin and catenins. Trends Cell Biol 15:216-221.

Takumi Y, Matsubara A, Rinvik E, Ottersen OP (1999) The arrangement of glutamate receptors in excitatory synapses. Ann N Y Acad Sci 868:474482.

van den Bout CJ, Machon O, Røsok Ø, Backman M, Krauss S (2002) The mouse enhancer element $D 6$ directs Cre recombinase activity in the neocortex and the hippocampus. Mech Dev 110:179-182.

von Bohlen und Halbach O (2010) Dendritic spine abnormalities in mental retardation. Cell Tissue Res 342:317-323.

Wu G, Malinow R, Cline HT (1996) Maturation of a central glutamatergic synapse. Science 274:972-976.

Yee ST, Okada Y, Ogasawara K, Omura S, Takatsuki A, Kakiuchi T, Muno D, Kominami E, Mizuochi T (1997) MHC class I presentation of an exogenous polypeptide antigen encoded by the murine AIDS defective virus. Microbiol Immunol 41:563-570.

Zennou V, Serguera C, Sarkis C, Colin P, Perret E, Mallet J, Charneau P (2001) The HIV-1 DNA flap stimulates HIV vector-mediated cell transduction in the brain. Nat Biotechnol 19:446-450.

Zhou Q, Homma KJ, Poo MM (2004) Shrinkage of dendritic spines associated with long-term depression of hippocampal synapses. Neuron 44:749-757.

Zhu JJ, Esteban JA, Hayashi Y, Malinow R (2000) Postnatal synaptic potentiation: delivery of GluR4-containing AMPA receptors by spontaneous activity. Nat Neurosci 3:1098-1106.

Zufferey R, Nagy D, Mandel RJ, Naldini L, Trono D (1997) Multiply attenuated lentiviral vector achieves efficient gene delivery in vivo. Nat Biotechnol 15:871-875. 